\title{
37. X-RAY MINERALOGY DATA, NORTHEASTERN PART OF THE INDIAN OCEAN, LEG 22, DEEP SEA DRILLING PROJECT ${ }^{1}$
}

\author{
John C. Matti, Ivar Zemmels, and Harry E. Cook, University of California, Riverside, California
}

\begin{abstract}
METHODS
Semiquantitative determinations of the mineral composition of bulk samples, $2-20 \mu \mathrm{m}$, and $<2 \mu \mathrm{m}$ fractions were performed according to the methods described in the reports of Legs 1 and 2 and in Appendix III, Volume IV. The mineral analyses of the $2-20 \mu \mathrm{m}$ and $<2 \mu \mathrm{m}$ fractions were performed on $\mathrm{CaCO}_{3}$-free residues.

The X-ray mineralogy results of this study are summarized in Tables 1 through 8 . The mineralogy data are presented in Tables 9 through 16 and in Figures 1 through 24. Sediment ages, lithologic units, and nomenclature of the sediment types in Figures 1 through 24 and Tables 1 through 8 are from the DSDP Leg 22 Hole Summaries and from a subsequent update supplied by Dr. A.C. Pimm, DSDP. The stratigraphic position of samples submitted for X-ray diffraction analysis from Leg 22 are listed in Tables 1 through 8 . The sample depth (in meters) below the sea floor in Tables 1 through 8 identifies the samples as they are reported in Tables 9 through 16 and Figures 1 through 24.

Results of X-ray diffraction analysis of squeezed residues for interstitial water studies at Site 218 are presented in Table 16 in the same format as other semiquantitative mineral data. The sample identification and the computed depth below the sea floor of the residues are given in Table 17. The squeezed residues were prepared for X-ray diffraction analysis by the same process used for all bulk $\mathrm{X}$-ray mineralogy samples, and the two sets of data should be compatible.

Several unidentified minerals were detected in Leg 22 samples. Their abundances were determined on a semiqualitative basis using a hypothetical mineral concentration factor of 3.0. Unidentified minerals are reported on a ranked, semiqualitative scale as outlined below:

Trace: $(>5 \%)$; diffraction pattern was weak and identification was made on the basis of two major diagnostic peaks.
\end{abstract}

\footnotetext{
${ }^{1}$ Institute of Geophysics and Planetary Physics, University of California, Riverside, California, Contribution No. 73-28.
}

Present: (5-25\%); a number of peaks of the mineral are visible in the diffraction pattern.

Abundant: (25-65\%); diffraction peaks of the mineral are prominent in the total diffraction pattern, but the peaks of other minerals are of an equivalent intensity.

Major: $(<65 \%)$; the diffraction peaks of the mineral dominate the diffraction pattern.

Although a certain quantity of the unidentified minerals is implied, their concentration is not included in the concentrations of the identified minerals, which are summed to $100 \%$.

On Leg 22 the use of drilling mud containing montmorillonite and barite was after Core 52 at Site 214 . There were no samples submitted for X-ray diffraction analysis which may have been exposed to drilling mud.

An unusual, expanding clay mineral was found to occur in rather pure form in the $<2 \mu \mathrm{m}$ fraction at the top of Unit 3 (Cores 42-52) at Site 214. The unit consists of a complex of lignite and volcanogenic sediments, mainly pyritic silty sand and clay-pebble conglomerate. The mineral was tentatively identified as beidellite, a silica-rich smectite. The (060) spacing was measured to be $1.491 \AA$ which differs from $1.500 \AA$ for montmorillonite (Brown, 1961). The 001 diffraction peaks are broad and weak whereas the hk0 peaks are sharp and strong. The concentration of this mineral diminishes with depth in the unit giving way to montmorillonite.

\section{ACKNOWLEDGMENTS}

The authors wish to acknowledge the excellent work of Nicki D. Coursey in the analysis and the quantification of X-ray diffraction data, of Paul D. Johnson in X-ray data acquisition and data processing, and of Tom W. Halverson, Jr., in sample preparation.

\section{REFERENCE}

Brown, G., 1961. The X-ray identification and crystal structure of clay minerals: London (Mineralogical Society). 
TABLE 1

Summary of X-Ray Mineralogy Samples, Sample Depths, Lithology, Age, and X-Ray Diffraction Results, Site 211

\begin{tabular}{|c|c|c|c|c|c|c|c|c|c|c|c|c|}
\hline \multirow{2}{*}{$\begin{array}{l}\text { Core, Section, } \\
\text { Depth in Section } \\
(\mathrm{cm})\end{array}$} & \multirow{2}{*}{$\begin{array}{l}\text { Sample } \\
\text { Depth Below } \\
\text { Sea Floor }(\mathrm{m})\end{array}$} & \multirow[b]{2}{*}{ Lithology } & \multirow[b]{2}{*}{ Age } & \multicolumn{3}{|c|}{$\begin{array}{c}\text { Bulk Sample } \\
\text { Major Constituent }\end{array}$} & \multicolumn{3}{|c|}{$\begin{array}{l}2-20 \mu \mathrm{m} \text { Fraction } \\
\text { Major Constituent }\end{array}$} & \multicolumn{3}{|c|}{$\begin{array}{c}<2 \mu \mathrm{m} \text { Fraction } \\
\text { Major Constituent }\end{array}$} \\
\hline & & & & 1 & 2 & 3 & 1 & 2 & 3 & 1 & 2 & 3 \\
\hline $\begin{array}{l}1-6,86-88 \\
3-1,90\end{array}$ & $\begin{array}{r}8.4 \\
57.9\end{array}$ & $\begin{array}{l}\text { Unit 1: Sili- } \\
\text { ceous, clay ooze } \\
\text { with volc. ash }\end{array}$ & $\begin{array}{l}\text { Quat. to } \\
\text { U. Plio. }\end{array}$ & $\begin{array}{l}\text { Quar. } \\
\text { Mica }\end{array}$ & $\begin{array}{l}\text { Mica } \\
\text { Mont. }\end{array}$ & $\begin{array}{l}\text { Mont. } \\
\text { Quar. }\end{array}$ & $\begin{array}{l}\text { Quar. } \\
\text { Quar. }\end{array}$ & $\begin{array}{l}\text { Plag. } \\
\text { Mica }\end{array}$ & $\begin{array}{l}\text { Mica } \\
\text { Plag. }\end{array}$ & $\begin{array}{l}\text { Mont. } \\
\text { Mont. }\end{array}$ & $\begin{array}{l}\text { Mica } \\
\text { Mica }\end{array}$ & $\begin{array}{l}\text { Kaol. } \\
\text { Kaol. }\end{array}$ \\
\hline $\begin{array}{l}4-2,70-72 \\
5-6,70-72 \\
6-6,105-107\end{array}$ & $\begin{array}{r}97.2 \\
141.2 \\
189.1\end{array}$ & $\begin{array}{l}\text { Unit } 2 \text { : Sili- } \\
\text { ceous fossil- } \\
\text { bearing, clay } \\
\text { ooze }\end{array}$ & Plio. & $\begin{array}{l}\text { Mica } \\
\text { Mont. } \\
\text { Mont. }\end{array}$ & $\begin{array}{l}\text { Quar. } \\
\text { Quar. } \\
\text { Quar. }\end{array}$ & $\begin{array}{l}\text { Mont. } \\
\text { Kaol. } \\
\text { Kaol. }\end{array}$ & $\begin{array}{l}\text { Quar. } \\
\text { Mont. } \\
\text { Mont. }\end{array}$ & $\begin{array}{l}\text { Mica } \\
\text { Quar. } \\
\text { Plag. . }\end{array}$ & $\begin{array}{l}\text { Plag. } \\
\text { Plag. } \\
\text { Quar. }\end{array}$ & $\begin{array}{l}\text { Mont. } \\
\text { Mont.. } \\
\text { Mont. }\end{array}$ & Quar. & Kaol.. \\
\hline $\begin{array}{l}10, \mathrm{CC} \\
11, \mathrm{CC}\end{array}$ & $\begin{array}{l}351.0 \\
398.0\end{array}$ & $\begin{array}{l}\text { Unit 4: Clays } \\
\text { and iron-oxide } \\
\text { and ash facies }\end{array}$ & (?) & $\begin{array}{l}\text { Mont. } \\
\text { K-Fe. }\end{array}$ & $\begin{array}{l}\text { Kaol. } \\
\text { Paly. }\end{array}$ & $\begin{array}{l}\text { Quar. } \\
\text { Mont. }\end{array}$ & $\begin{array}{l}\text { Insuffi } \\
\mathrm{K}-\mathrm{Fe} \text {. }\end{array}$ & $\begin{array}{l}\text { ient resi } \\
\text { Quar. }\end{array}$ & $\begin{array}{l}\text { Iue } \\
\text { Mica }\end{array}$ & $\begin{array}{l}\text { Mont. } \\
\text { Mont. }\end{array}$ & $\begin{array}{l}\text { Kaol. } \\
\text { K-Fe. }\end{array}$ & $\begin{array}{l}\text { Mica. } \\
\text { Paly. }\end{array}$ \\
\hline $\begin{array}{l}12-1,13-14 \\
12-2,125-127 \\
12-2,138-140 \\
13-1,63-64 \\
13-1,147-148 \\
14-1,59-61\end{array}$ & $\begin{array}{l}409.1 \\
411.8 \\
411.9 \\
418.6 \\
419.5 \\
428.1\end{array}$ & $\begin{array}{l}\text { Unit 6: } \\
\text { Variegated, } \\
\text { nanno ooze } \\
\text { and clay }\end{array}$ & $\begin{array}{l}\text { Basal Cam- } \\
\text { panian to } \\
\text { Basal Maas- } \\
\text { trichtian }\end{array}$ & $\begin{array}{l}\text { Paly. } \\
\text { Calc. } \\
\text { Calc. } \\
\text { Calc. } \\
\text { Paly. } \\
\text { Paly. }\end{array}$ & $\begin{array}{l}\text { Calc. } \\
\text { K-Fe. } \\
\text { Paly. } \\
\text { Calc. } \\
\text { Quar. }\end{array}$ & $\begin{array}{l}\text { K-Fe. } \\
\text { Quar. } \\
\text { K-Fe. } \\
\text { K-Fe. }\end{array}$ & $\begin{array}{l}\mathrm{K}-\mathrm{Fe} . \\
\mathrm{K}-\mathrm{Fe} . \\
\mathrm{K}-\mathrm{Fe} . \\
\mathrm{K}-\mathrm{Fe} . \\
\text { Quar. } \\
\text { Quar. }\end{array}$ & $\begin{array}{l}\text { Quar. } \\
\text { Quar. } \\
\text { Quar. } \\
\text { Quar. } \\
\text { K-Fe. } \\
\text { K-Fe. }\end{array}$ & $\begin{array}{l}\text { Mica. } \\
\text { Mica. } \\
\text { Mica. } \\
\text { Mica. }\end{array}$ & $\begin{array}{l}\text { Paly. } \\
\text { Mica. } \\
\text { Mont. } \\
\text { Mont. } \\
\text { Paly. } \\
\text { Paly. }\end{array}$ & $\begin{array}{l}\text { Mont. } \\
\text { K-Fe. } \\
\text { Mica } \\
\text { Paly. } \\
\text { Mont. } \\
\text { K-Fe. }\end{array}$ & $\begin{array}{l}\text { K-Fe. } \\
\text { Hema. } \\
\text { K-Fe. } \\
\text { Mica. } \\
\text { K-Fe. } \\
\text { Quar. }\end{array}$ \\
\hline
\end{tabular}

TABLE 2

Summary of X-Ray Mineralogy Samples, Sample Depths, Lithology, Age, and X-Ray Diffraction Results, Site 212

\begin{tabular}{|c|c|c|c|c|c|c|c|c|c|c|}
\hline \multirow{2}{*}{$\begin{array}{l}\text { Core, Section } \\
\text { Depth in Section } \\
(\mathrm{cm})\end{array}$} & \multirow{2}{*}{$\begin{array}{l}\text { Sample } \\
\text { Depth Below } \\
\text { Sea Floor }(\mathrm{m})\end{array}$} & \multirow[b]{2}{*}{ Lithology } & \multirow[b]{2}{*}{ Age } & \multicolumn{3}{|c|}{$\begin{array}{c}\text { Bulk Sample } \\
\text { Major Constituent }\end{array}$} & $\begin{array}{l}\text { 2-20 } \mu \mathrm{m} \text { Fraction } \\
\text { Major Constituent }\end{array}$ & \multicolumn{3}{|c|}{$\begin{array}{c}<2 \mu \text { m Fraction } \\
\text { Major Constituent }\end{array}$} \\
\hline & & & & 1 & 2 & 3 & $\begin{array}{lll}1 & 2 & 3\end{array}$ & 1 & 2 & 3 \\
\hline $2-1,121-123$ & 10.7 & $\begin{array}{l}\text { Unit 1: } \\
\text { Interbedded } \\
\text { nanno ooze } \\
\text { and clay }\end{array}$ & Mid. Plio. & Quar. & Mica. & Kaol. & Insufficient residue & Mont. & Kaol. & Quar. \\
\hline $\begin{array}{l}10-6,78-80 \\
12-5,137-138\end{array}$ & $\begin{array}{l}172.8 \\
228.9\end{array}$ & $\begin{array}{l}\text { Unit 2: } \\
\text { Nanno ooze } \\
\text { and chalk } \\
\text { with minor } \\
\text { clay }\end{array}$ & $\begin{array}{l}\text { Mid. and } \\
\text { L. Mioc. }\end{array}$ & $\begin{array}{l}\text { Calc. } \\
\text { Calc. }\end{array}$ & & & Insufficient residue & $\begin{array}{l}\text { Mont. } \\
\text { Mont. }\end{array}$ & $\begin{array}{l}\text { Kaol. } \\
\text { Kaol. }\end{array}$ & $\begin{array}{l}\text { Mica. } \\
\text { Mica. }\end{array}$ \\
\hline $\begin{array}{l}15-1,106-109 \\
16-1\end{array}$ & $\begin{array}{l}289.1 \\
297.5\end{array}$ & $\begin{array}{l}\text { Unit 3: } \\
\text { Claystone }\end{array}$ & (?) & $\begin{array}{l}\text { Quar. } \\
\text { Quar. }\end{array}$ & $\begin{array}{l}\text { Mica. } \\
\text { Mont. }\end{array}$ & $\begin{array}{l}\mathrm{K}-\mathrm{Fe} . \\
\text { Kaol. }\end{array}$ & $\begin{array}{l}\text { Insufficient residue } \\
\text { Quar. Mica. K-Fe. }\end{array}$ & $\begin{array}{l}\text { Mont. } \\
\text { Mont. }\end{array}$ & $\begin{array}{l}\text { Paly. } \\
\text { Kaol. }\end{array}$ & $\begin{array}{l}\text { Kaol. } \\
\text { Quar. }\end{array}$ \\
\hline $18-3,20-22$ & 319.7 & $\begin{array}{l}\text { Unit } 4 \text { : } \\
\text { Nanno chalk }\end{array}$ & M. Eoc. & Clin. & Paly. & Quar. & Clin. Phil. Quar. & Mont. & Paly. & $\mathrm{K}-\mathrm{Fe}$. \\
\hline $\begin{array}{l}27-1,104-106 \\
27-1,130-132 \\
27-4,6-7 \\
28-1,16-18 \\
29-1,29-31\end{array}$ & $\begin{array}{l}403.0 \\
403.3 \\
406.6 \\
411.7 \\
421.3\end{array}$ & $\begin{array}{l}\text { Unit 5: } \\
\text { Claystone }\end{array}$ & (?) & $\begin{array}{l}\text { Mont. } \\
\text { Mont. } \\
\text { Mont. } \\
\text { Kaol. } \\
\text { Kaol. }\end{array}$ & $\begin{array}{l}\text { Mica. } \\
\text { Quar. } \\
\text { Kaol. } \\
\text { Mica. } \\
\text { Mica. }\end{array}$ & $\begin{array}{l}\text { Kaol. } \\
\text { Kaol. } \\
\text { Quar. } \\
\text { K-Fe. } \\
\text { K-Fe. }\end{array}$ & $\begin{array}{l}\text { Quar. Mica. K-Fe. } \\
\text { Insufficient residue }\end{array}$ & $\begin{array}{l}\text { Mont. } \\
\text { Mont. } \\
\text { Mont. } \\
\text { Mont. } \\
\text { Mont. }\end{array}$ & $\begin{array}{l}\text { Kaol. } \\
\text { Kaol. } \\
\text { Kaol. } \\
\text { Kaol. } \\
\text { Kaol. }\end{array}$ & $\begin{array}{l}\text { Quar. } \\
\text { Quar. } \\
\text { Quar. } \\
\text { Mica. } \\
\text { Mica. }\end{array}$ \\
\hline $\begin{array}{l}32-6,45-46 \\
35-3,84-86 \\
35-4,84-86\end{array}$ & $\begin{array}{l}457.5 \\
481.8 \\
483.3\end{array}$ & $\begin{array}{l}\text { Unit } 6: \\
\text { Nanno chalk }\end{array}$ & $\begin{array}{l}\text { Late } \\
\text { Cret. }\end{array}$ & $\begin{array}{l}\text { Calc. } \\
\text { Calc. } \\
\text { Calc. }\end{array}$ & & & $\begin{array}{l}\text { Quar. Clin. Plag. } \\
\text { Insufficient residue }\end{array}$ & $\begin{array}{l}\text { Mont. } \\
\text { Mont. } \\
\text { Mont. }\end{array}$ & & \\
\hline $\begin{array}{l}35-5,12-14 \\
35-5,42-46 \\
36-1,109-110 \\
37-1,26-27 \\
38-1,72-73 \\
38-2,20-21\end{array}$ & $\begin{array}{l}484.1 \\
484.4 \\
488.6 \\
497.3 \\
507.2 \\
508.2\end{array}$ & $\begin{array}{l}\text { Unit } 7: \\
\text { Claystone }\end{array}$ & (?) & $\begin{array}{l}\text { Mont. } \\
\text { Mont. } \\
\text { Paly. } \\
\text { Paly. } \\
\text { Paly. } \\
\text { Paly. }\end{array}$ & $\begin{array}{l}\text { Mica. } \\
\text { Paly. } \\
\text { Quar. } \\
\text { Quar. } \\
\text { Clin. } \\
\text { K-Fe. }\end{array}$ & $\begin{array}{l}\text { Quar. } \\
\text { Quar. } \\
\text { K-Fe. } \\
\text { K-Fe. } \\
\text { Quar. } \\
\text { Quar. }\end{array}$ & $\begin{array}{lll}\text { Quar. } & \text { K-Fe. } & \text { Plag. } \\
\text { Quar. } & \text { K-Fe. } & \text { Plag. } \\
\text { Quar. } & \text { K-Fe. } & \text { Plag. } \\
\text { Insufficient residue } \\
\text { Clin. } & \text { Quar. } & \text { Mica. } \\
\text { K-Fe. } & \text { Quar. } & \text { Mica. }\end{array}$ & $\begin{array}{l}\text { Mont. } \\
\text { Mont. } \\
\text { Paly. } \\
\text { Paly. } \\
\text { Paly. } \\
\text { Mont. }\end{array}$ & $\begin{array}{l}\text { Paly. } \\
\text { Paly. } \\
\text { Quar. } \\
\text { Quar. } \\
\text { Clin. } \\
\text { Paly. }\end{array}$ & $\begin{array}{l}\text { Quar. } \\
\text { Quar. } \\
\text { K-Fe. } \\
\text { Mont. } \\
\text { Mica. } \\
\text { K-Fe. }\end{array}$ \\
\hline
\end{tabular}


TABLE 3

Summary of X-Ray Mineralogy Samples, Sample Depths, Lithology, Age, and X-Ray Diffraction Results, Site 213

\begin{tabular}{|c|c|c|c|c|c|c|c|c|c|c|c|c|}
\hline \multirow{2}{*}{$\begin{array}{l}\text { Core, Section, } \\
\text { Depth in Section } \\
(\mathrm{cm})\end{array}$} & \multirow{2}{*}{$\begin{array}{c}\text { Sample } \\
\text { Depth Below } \\
\text { Sea Floor }(\mathrm{m})\end{array}$} & \multirow[b]{2}{*}{ Lithology } & \multirow[b]{2}{*}{ Age } & \multicolumn{3}{|c|}{$\begin{array}{c}\text { Bulk Sample } \\
\text { Major Constituent }\end{array}$} & \multicolumn{3}{|c|}{$\begin{array}{l}2-20 \mu \mathrm{m} \text { Fraction } \\
\text { Major Constituent }\end{array}$} & \multicolumn{3}{|c|}{$\begin{array}{l}<2 \mu \mathrm{m} \text { Fraction } \\
\text { Major Constituent }\end{array}$} \\
\hline & & & & 1 & 2 & 3 & 1 & 2 & 3 & 1 & 2 & 3 \\
\hline $\begin{array}{l}1-1,7-9 \\
1-1,72-74 \\
1-4,130-132 \\
2-5,31-33 \\
2-5,51-53 \\
3-6,13-15 \\
6-5,70-72 \\
8-3,12-14 \\
8-6,142-144\end{array}$ & $\begin{array}{r}0.1 \\
0.7 \\
5.8 \\
15.3 \\
15.5 \\
26.1 \\
53.7 \\
69.1 \\
74.9\end{array}$ & $\begin{array}{l}\text { Unit 1: } \\
\text { Radiolarian } \\
\text { and diatom } \\
\text { ooze with } \\
\text { varying clay } \\
\text { content }\end{array}$ & $\begin{array}{l}\text { Upper } \\
\text { Miocene } \\
\text { through } \\
\text { Quaternary }\end{array}$ & $\begin{array}{l}\text { Quar. } \\
\text { Quar. } \\
\text { Quar. } \\
\text { Quar. } \\
\text { Quar. } \\
\text { Mica. } \\
\text { Quar. } \\
\text { Quar. } \\
\text { Mont. }\end{array}$ & $\begin{array}{l}\text { Mica } \\
\text { Mica } \\
\text { Mica } \\
\text { Mica } \\
\text { Mica } \\
\text { Quar. } \\
\text { Mica } \\
\text { Mont. } \\
\text { Quar. }\end{array}$ & $\begin{array}{l}\text { Mont. } \\
\text { Kaol. } \\
\text { Kaol. } \\
\text { Kaol. } \\
\text { Kaol. } \\
\text { Kaol. } \\
\text { Kaol. } \\
\text { Kaol. } \\
\text { Mica }\end{array}$ & Insuf & icient re & sidue & $\begin{array}{l}\text { Mont. } \\
\text { Mont. } \\
\text { Quar. } \\
\text { Mont. } \\
\text { Mont. } \\
\text { Mont. } \\
\text { Mont. } \\
\text { Mont. } \\
\text { Mont. }\end{array}$ & $\begin{array}{l}\text { Quar. } \\
\text { Quar. } \\
\text { Kaol. } \\
\text { Kaol. } \\
\text { Quar. } \\
\text { Kaol. } \\
\text { Kaol. } \\
\text { Kaol. } \\
\text { Quar. }\end{array}$ & $\begin{array}{l}\text { Kaol. } \\
\text { Mica } \\
\text { Mont. } \\
\text { Quar. } \\
\text { Kaol. } \\
\text { Mica } \\
\text { Quar. } \\
\text { Quar. } \\
\text { Kaol. }\end{array}$ \\
\hline $\begin{array}{r}9-6,82-84 \\
10-3,72-74 \\
11-4,70-72 \\
13-3,70-72 \\
14-2,72-74\end{array}$ & $\begin{array}{r}89.2 \\
88.7 \\
99.7 \\
117.2 \\
125.2\end{array}$ & $\begin{array}{l}\text { Unit 2: } \\
\text { Zeolitic } \\
\text { clay }\end{array}$ & Mid. Mioc. & $\begin{array}{l}\text { Mont. } \\
\text { Mont. } \\
\text { Phil. } \\
\text { Phil. } \\
\text { Paly. }\end{array}$ & $\begin{array}{l}\text { Quar. } \\
\text { Phil. } \\
\text { Mont. } \\
\text { Paly. } \\
\text { K-Fe. }\end{array}$ & $\begin{array}{l}\text { Mica } \\
\text { Kaol. } \\
\text { Paly. } \\
\text { K-Fe. } \\
\text { Mica. }\end{array}$ & $\begin{array}{l}\text { Insuf } \\
\text { Phil. } \\
\text { Phil. } \\
\text { Phil. } \\
\text { K-Fe. }\end{array}$ & $\begin{array}{l}\text { icient re } \\
\text { Quar. } \\
\text { Quar. } \\
\text { Quar. }\end{array}$ & $\begin{array}{l}\text { idue } \\
\text { Mont. } \\
\text { Paly. } \\
\text { Paly. }\end{array}$ & $\begin{array}{l}\text { Mont. } \\
\text { Mont. } \\
\text { Mont. } \\
\text { Phil. } \\
\text { Mont. }\end{array}$ & $\begin{array}{l}\text { Kaol. } \\
\text { Quar. } \\
\text { Paly. } \\
\text { Mont. } \\
\text { Paly. }\end{array}$ & $\begin{array}{l}\text { Kaol. } \\
\text { Kaol. } \\
\text { Quar. } \\
\text { K-Fe. }\end{array}$ \\
\hline $16-2,112-114$ & 144.6 & $\begin{array}{l}\text { Unit 3: } \\
\text { Nanno ooze }\end{array}$ & a & Calc. & & & Quar. & $\mathrm{K}-\mathrm{Fe}$. & & $\mathrm{U}-2$ & & \\
\hline $16-4,142-144$ & 147.9 & $\begin{array}{l}\text { Unit } 4 \text { : } \\
\text { Iron oxide and } \\
\text { manganese } \\
\text { facies }\end{array}$ & (?) & Paly. & $\mathrm{K}-\mathrm{Fe}$. & Calc. & $\mathrm{K}-\mathrm{Fe}$. & Quar. & Mica & Paly. & $\mathrm{K}-\mathrm{Fe}$. & Mont. \\
\hline
\end{tabular}

aUnit 3 is early Eocene and late Paleocere in age.

TABLE 4

Summary of X-Ray Mineralogy Samples, Sample Depths, Lithology, Age, and X-Ray Diffraction Results, Site 214

\begin{tabular}{|c|c|c|c|c|c|c|c|c|c|c|c|c|}
\hline \multirow{2}{*}{$\begin{array}{l}\text { Core, Section, } \\
\text { Depth in Section } \\
(\mathrm{cm})\end{array}$} & \multirow{2}{*}{$\begin{array}{l}\text { Sample } \\
\text { Depth Below } \\
\text { Sea Floor }(\mathrm{m})\end{array}$} & \multirow[b]{2}{*}{ Lithology } & \multirow[b]{2}{*}{ Age } & \multicolumn{3}{|c|}{$\begin{array}{c}\text { Bulk Sample } \\
\text { Major Constituent }\end{array}$} & \multicolumn{3}{|c|}{$\begin{array}{l}2-20 \mu \mathrm{m} \text { Fraction } \\
\text { Major Constituent }\end{array}$} & \multicolumn{3}{|c|}{$\begin{array}{l}<2 \mu \mathrm{m} \text { Fraction } \\
\text { Major Constituent }\end{array}$} \\
\hline & & & & 1 & 2 & 3 & 1 & 2 & 3 & 1 & 2 & 3 \\
\hline $\begin{array}{l}3-1,28-30 \\
3-3,135-137\end{array}$ & $\begin{array}{l}19.3 \\
23.4\end{array}$ & $\begin{array}{l}\text { Unit 1: } \\
\text { Foram and } \\
\text { nanno ooze }\end{array}$ & a & $\begin{array}{l}\text { Calc. } \\
\text { Calc. }\end{array}$ & & & Insuf & cient res & idue & $\begin{array}{l}\text { Mont. } \\
\text { Quar. }\end{array}$ & $\begin{array}{l}\text { Quar. } \\
\text { Mica }\end{array}$ & $\begin{array}{l}\text { Kaol. } \\
\text { Kaol. }\end{array}$ \\
\hline $\begin{array}{l}36-2,10-12 \\
36-3,54-56 \\
37-2,120-122 \\
38-4,0\end{array}$ & $\begin{array}{l}334.6 \\
336.5 \\
345.2 \\
356.5\end{array}$ & $\begin{array}{l}\text { Unit 2a: } \\
\text { Glauconitic } \\
\text { carbonate silt } \\
\text { and sand }\end{array}$ & Paleocene & $\begin{array}{l}\text { Plag. } \\
\text { Calc. } \\
\text { Calc. } \\
\text { Calc. }\end{array}$ & $\begin{array}{l}\text { Mont. } \\
\text { Clin. } \\
\text { Plag. } \\
\text { Plag. }\end{array}$ & $\begin{array}{l}\text { Apat. } \\
\text { Plag. } \\
\text { Mont. }\end{array}$ & $\begin{array}{l}\text { Plag. } \\
\text { Clin. } \\
\text { Plag. } \\
\text { Mont. }\end{array}$ & $\begin{array}{l}\text { Mont. } \\
\text { Plag. } \\
\text { Pyri. } \\
\text { Plag. }\end{array}$ & Pyri. & $\begin{array}{l}\text { Mont. } \\
\text { Mont. } \\
\text { Mont. } \\
\text { Mont. }\end{array}$ & $\begin{array}{l}\text { Plag. } \\
\text { Plag. } \\
\text { Plag. } \\
\text { Pyri. }\end{array}$ & Apat. \\
\hline $\begin{array}{l}40-1,108-110 \\
41-3,98-100 \\
41-3,134-136\end{array}$ & $\begin{array}{l}372.1 \\
384.5 \\
384.8\end{array}$ & $\begin{array}{l}\text { Unit } 2 \mathrm{~b} \text { : } \\
\text { Glauconitic } \\
\text { shelly car- } \\
\text { bonate silt }\end{array}$ & Paleocene & $\begin{array}{l}\text { Plag. } \\
\text { Plag. } \\
\text { Mont. }\end{array}$ & $\begin{array}{l}\text { Calc. } \\
\text { Pyri. } \\
\text { Plag. }\end{array}$ & $\begin{array}{l}\text { Mont. } \\
\text { Mont. } \\
\text { Pyri. }\end{array}$ & $\begin{array}{l}\text { Plag. } \\
\text { Pyri. } \\
\text { Pyri. }\end{array}$ & $\begin{array}{l}\text { Mont. } \\
\text { Plag. } \\
\text { Plag. }\end{array}$ & $\begin{array}{l}\text { Pyri. } \\
\text { Mont. }\end{array}$ & $\begin{array}{l}\text { Mont. } \\
\text { Mont. } \\
\text { Mont. }\end{array}$ & Plag. & \\
\hline $\begin{array}{l}42-1,93-95 \\
42-1,108-110 \\
44-1,93-95 \\
44-1,110-112 \\
46-2,27-29 \\
46-2,143-145 \\
52-1,126\end{array}$ & $\begin{array}{l}390.9 \\
391.1 \\
403.4 \\
403.6 \\
423.3 \\
424.4 \\
477.8\end{array}$ & $\begin{array}{l}\text { Unit 3: } \\
\text { Lignite, volc. } \\
\text { clay, tuff, and } \\
\text { lapilli tuff }\end{array}$ & (?) & $\begin{array}{l}\text { Clin. } \\
\text { Clin. } \\
\text { Mont. } \\
\text { K-Fe. } \\
\text { K-Fe. } \\
\text { Mont. } \\
\text { Mont. }\end{array}$ & $\begin{array}{l}\text { K-Fe. } \\
\text { Kaol. } \\
\text { Mont. } \\
\text { K-Fe. } \\
\text { K-Fe. }\end{array}$ & $\begin{array}{l}\text { Pyri. } \\
\text { Quar. } \\
\text { Quar. } \\
\text { Pyri. }\end{array}$ & $\begin{array}{l}\text { Clin. } \\
\text { Clin. } \\
\text { K-Fe. } \\
\text { K-Fe. } \\
\text { K-Fe. } \\
\text { K-Fe. } \\
\text { K-Fe. }\end{array}$ & $\begin{array}{l}\text { K-Fe. } \\
\text { Mont. } \\
\text { Quar. } \\
\text { Quar. } \\
\text { Pyri. } \\
\text { Mont. }\end{array}$ & $\begin{array}{l}\text { Quar. } \\
\text { Pyri. } \\
\text { Pyri. } \\
\text { Mont. } \\
\text { Side. }\end{array}$ & $\begin{array}{l}\text { Beid. } \\
\text { Clin. } \\
\text { Mont. } \\
\text { Mica } \\
\text { Mont. } \\
\text { Mont. } \\
\text { Mont. }\end{array}$ & $\begin{array}{l}\text { Pyri. } \\
\text { Kaol. } \\
\text { K-Fe. } \\
\text { Mica }\end{array}$ & $\begin{array}{l}\text { Beid. } \\
\text { K-Fe. } \\
\text { U-3 }\end{array}$ \\
\hline
\end{tabular}

${ }^{\text {a Core }} 3$ is Pleistocene or latest Pliocene in age. 
TABLE 5

Summary of X-Ray Mineralogy Samples, Sample Depths, Lithology, Age, and X-Ray Diffraction Results, Site 215

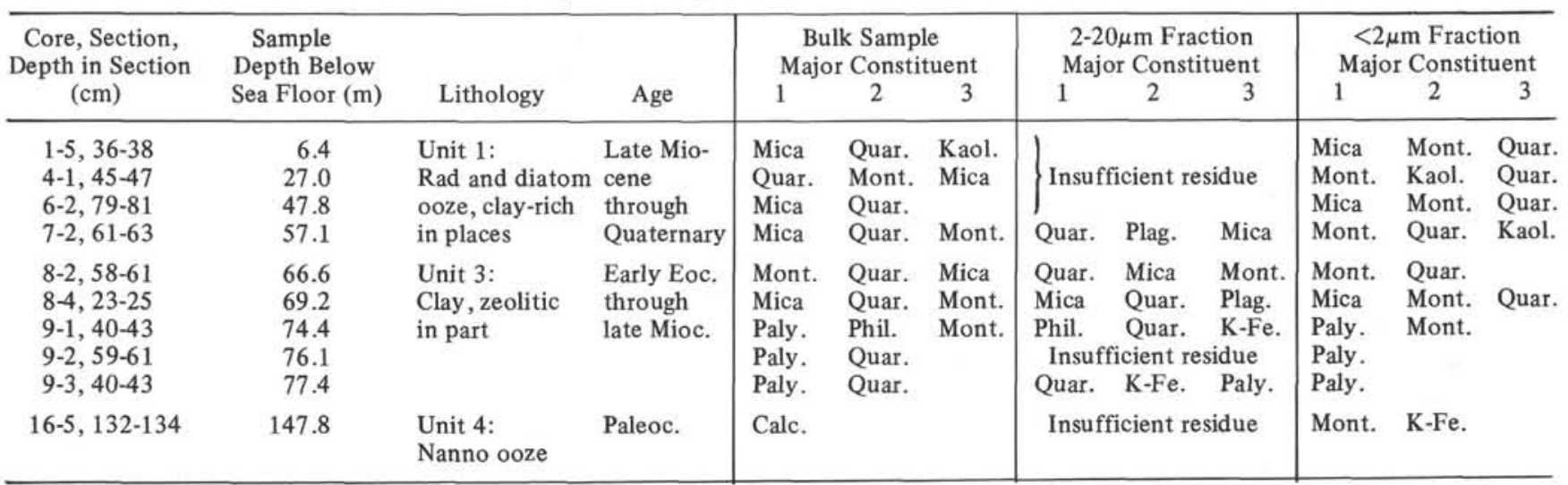

TABLE 6

Summary of X-Ray Mineralogy Samples, Sample Depths, Lithology Age, and X-Ray Diffraction Results, Site 216

\begin{tabular}{|c|c|c|c|c|c|c|c|c|c|c|c|c|}
\hline \multirow{2}{*}{$\begin{array}{l}\text { Core, Section } \\
\text { Depth in Section } \\
(\mathrm{cm})\end{array}$} & \multirow{2}{*}{$\begin{array}{c}\text { Sample } \\
\text { Depth Below } \\
\text { Sea Floor (m) }\end{array}$} & \multirow[b]{2}{*}{ Lithology } & \multirow[b]{2}{*}{ Age } & \multicolumn{3}{|c|}{$\begin{array}{c}\text { Bulk Sample } \\
\text { Major Constituent }\end{array}$} & \multicolumn{3}{|c|}{$\begin{array}{l}2-20 \mu \mathrm{m} \text { Fraction } \\
\text { Major Constituent }\end{array}$} & \multicolumn{3}{|c|}{$\begin{array}{l}<2 \mu \mathrm{m} \text { Fraction } \\
\text { Major Constituent }\end{array}$} \\
\hline & & & & 1 & 2 & 3 & 1 & 2 & 3 & 1 & 2 & 3 \\
\hline $4-1,60-62$ & 121.1 & $\begin{array}{l}\text { Unit 1a: } \\
\text { Nanno ooze }\end{array}$ & a & Calc. & & & Insuff & icient re & idue & Paly. & Kaol. & Mica. \\
\hline $\begin{array}{l}19-1,109-110 \\
21-2,67-68\end{array}$ & $\begin{array}{l}292.6 \\
311.2\end{array}$ & $\begin{array}{l}\text { Unit } 1 \mathrm{~b} \text { : } \\
\text { Nanno chalk }\end{array}$ & & $\begin{array}{l}\text { Calc. } \\
\text { Calc. }\end{array}$ & Paly. & & Insuff & cient re & idue & $\begin{array}{l}\text { Paly. } \\
\text { Paly. }\end{array}$ & Mont. & \\
\hline $24-5,92-94$ & 345.9 & Unit $1 c^{c}$ & c & Calc. & & & Clin. & Mont. & & Mont. & Paly. & \\
\hline $\begin{array}{l}25-4,91-93 \\
26-2,70-72\end{array}$ & $\begin{array}{l}353.9 \\
360.2\end{array}$ & Unit $2 \mathrm{a}^{\mathrm{d}}$ & d & $\begin{array}{l}\text { Calc. } \\
\text { Mont. }\end{array}$ & $\begin{array}{l}\text { Mont. } \\
\text { Calc. }\end{array}$ & Anal. & $\begin{array}{l}\text { Mont. } \\
\text { Mont. }\end{array}$ & $\begin{array}{l}\text { Plag. } \\
\text { Anal. }\end{array}$ & Clin. & $\begin{array}{l}\text { Mont. } \\
\text { Mont. }\end{array}$ & & \\
\hline $\begin{array}{l}30-1,88 \\
31-1,110-111 \\
32-3,2-3 \\
33-1,97-99 \\
34-1,53-55 \\
34-4,67-68 \\
34-4,71-72\end{array}$ & $\begin{array}{l}396.9 \\
406.6 \\
418.0 \\
425.5 \\
434.5 \\
439.2 \\
439.2\end{array}$ & $\begin{array}{l}\text { Unit } 2 \mathrm{~b} \text { : } \\
\text { Intermixed } \\
\text { volcanic clay } \\
\text { and micarb } \\
\text { chalk with } \\
\text { discrete beds } \\
\text { of ash }\end{array}$ & $\begin{array}{l}\text { Late } \\
\text { Maas- } \\
\text { trichtian }\end{array}$ & $\begin{array}{l}\text { Calc. } \\
\text { Calc. } \\
\text { Calc. } \\
\text { Mont. } \\
\text { Mont. } \\
\text { Calc. } \\
\text { Mica }\end{array}$ & $\begin{array}{l}\text { Augi. } \\
\text { Plag. } \\
\text { Phil. } \\
\text { Calc. } \\
\text { Phil. } \\
\text { Phil. }\end{array}$ & $\begin{array}{l}\text { Plag. } \\
\text { Augi. } \\
\text { Calc. } \\
\text { Clin. } \\
\text { Mont. } \\
\text { Mont. }\end{array}$ & $\begin{array}{l}\text { Augi. } \\
\text { Augi. } \\
\text { Augi. } \\
\text { Mont. } \\
\text { Mont. } \\
\text { Phil. } \\
\text { Phil. }\end{array}$ & $\begin{array}{l}\text { Plag. } \\
\text { Plag. } \\
\text { Plag. } \\
\text { Phil. } \\
\text { Plag. } \\
\text { Mont. } \\
\text { Mica }\end{array}$ & $\begin{array}{l}\text { Pyri. } \\
\text { Pyri. } \\
\text { Mont. } \\
\text { Augi. } \\
\text { Clin. } \\
\text { Mont. }\end{array}$ & $\begin{array}{l}\text { Mont. } \\
\text { Mont. } \\
\text { Mont. } \\
\text { Mont. } \\
\text { Mont. } \\
\text { Mont. } \\
\text { Mica }\end{array}$ & $\begin{array}{l}\text { Augi. } \\
\text { Augi. } \\
\text { Pyri. }\end{array}$ & $\begin{array}{l}\text { Plag. } \\
\text { Pyri. }\end{array}$ \\
\hline
\end{tabular}

aUnit 1a is Pleistocene to late Oligocene in age.

bUnit $1 \mathrm{~b}$ is late Oligocene to Paleocene (Danian) in age.

cUnit 1c consists of glauconite-bearing, clay-rich, micarb chalk, and is late Maastrichtian in age.

dUnit 2a consists of glauconitic, volcanic, clay, micarb chalk, and is late Maastrichtian in age.

TABLE 7

Summary of X-Ray Mineralogy Samples, Sample Depths, Lithology, Age, and X-Ray Diffraction Results, Site 217

\begin{tabular}{|c|c|c|c|c|c|c|c|c|c|c|}
\hline \multirow{2}{*}{$\begin{array}{c}\text { Core, Section } \\
\text { Depth in Section } \\
(\mathrm{cm})\end{array}$} & \multirow{2}{*}{$\begin{array}{c}\text { Sample } \\
\text { Depth Below } \\
\text { Sea Floor }(\mathrm{m})\end{array}$} & \multirow[b]{2}{*}{ Lithology } & \multirow[b]{2}{*}{ Age } & \multicolumn{3}{|c|}{$\begin{array}{c}\text { Bulk Sample } \\
\text { Major Constituent }\end{array}$} & $\begin{array}{c}2-2 \mu \mathrm{m} \text { Fraction } \\
\text { Major Constituent }\end{array}$ & \multicolumn{3}{|c|}{$\begin{array}{c}<2 \mu \text { Fraction } \\
\text { Major Constituent }\end{array}$} \\
\hline & & & & 1 & 2 & 3 & $\begin{array}{lll}1 & 2 & 3 \\
\end{array}$ & 1 & 2 & 3 \\
\hline $\begin{array}{l}1-1,40-42 \\
2-3,70-72 \\
4-5,62-64\end{array}$ & $\begin{array}{r}0.4 \\
43.7 \\
122.6\end{array}$ & $\begin{array}{l}\text { Unit 1: } \\
\text { Clay nanno- } \\
\text { fossil ooze }\end{array}$ & $\begin{array}{l}\text { Recent } \\
\text { to late } \\
\text { Miocene }\end{array}$ & $\begin{array}{l}\text { Calc. } \\
\text { Calc. } \\
\text { Calc. }\end{array}$ & Mica & Quar. & $\begin{array}{l}\text { Insufficient residue } \\
\text { Quar. Mica Plag. } \\
\text { Insufficient residue }\end{array}$ & $\begin{array}{l}\text { Mica } \\
\text { Mont. } \\
\text { Mont. }\end{array}$ & $\begin{array}{l}\text { Mont. } \\
\text { Mica. } \\
\text { Mica }\end{array}$ & $\begin{array}{l}\text { Quar. } \\
\text { Quar. } \\
\text { Kaol. }\end{array}$ \\
\hline $34-1,45-46$ & 582.5 & Unit $2 \mathrm{da}^{\mathrm{a}}$ & a & Calc. & Mont. & & Mont. & Mont. & & \\
\hline $37-1,125-126$ & 611.8 & $\begin{array}{l}\text { Unit 3: } \\
\text { Dolarenite, } \\
\text { chert, and } \\
\text { claystone }\end{array}$ & $\begin{array}{l}\text { Late } \\
\text { Campanian }\end{array}$ & Dolo. & Calc. & Mont. & Quar. Bari. & Mont. & & \\
\hline
\end{tabular}

aUnit $2 \mathrm{~d}$ is middle Maastrichtian to late Campanian in age and consists of micarb chalk, partly shelly, and chert. 
TABLE 8

Summary of X-Ray Mineralogy Samples, Sample Depths, Lithology, Age, and X-Ray Diffraction Results, Site 218

\begin{tabular}{|c|c|c|c|c|c|c|c|c|c|c|c|c|}
\hline \multirow{2}{*}{$\begin{array}{l}\text { Core, Section, } \\
\text { Depth in Section } \\
(\mathrm{cm})\end{array}$} & \multirow{2}{*}{$\begin{array}{c}\text { Sample } \\
\text { Depth Below } \\
\text { Sea Floor }(\mathrm{m})\end{array}$} & \multirow[b]{2}{*}{ Lithology } & \multirow[b]{2}{*}{ Age } & \multicolumn{3}{|c|}{$\begin{array}{c}\text { Bulk Sample } \\
\text { Major Constituent }\end{array}$} & \multicolumn{3}{|c|}{$\begin{array}{c}2-2 \mu \mathrm{m} \text { Fraction } \\
\text { Major Constituent }\end{array}$} & \multicolumn{3}{|c|}{$\begin{array}{c}<2 \mu \text { Fraction } \\
\text { Major Constituent }\end{array}$} \\
\hline & & & & 1 & 2 & 3 & 1 & 2 & 3 & 1 & 2 & 3 \\
\hline $\begin{array}{l}2-1,128-130 \\
2-3,120-122\end{array}$ & $\begin{array}{l}5.3 \\
8.2\end{array}$ & $\begin{array}{l}\text { Unit } 1 \text { : } \\
\text { Clay and silt- } \\
\text { rich nanno } \\
\text { ooze }\end{array}$ & Quat. & $\begin{array}{l}\text { Mica } \\
\text { Mica }\end{array}$ & $\begin{array}{l}\text { Quar. } \\
\text { Quar. }\end{array}$ & Calc. & $\begin{array}{l}\text { Mica } \\
\text { Mica }\end{array}$ & $\begin{array}{l}\text { Quar. } \\
\text { Quar. }\end{array}$ & $\begin{array}{l}\text { Plag. } \\
\text { Plag. }\end{array}$ & $\begin{array}{l}\text { Mica. } \\
\text { Mica }\end{array}$ & $\begin{array}{l}\text { Mont. } \\
\text { Quar. }\end{array}$ & Quar. \\
\hline $\begin{array}{l}5-1,103-105 \\
6-2,120-122 \\
8-2,63-65\end{array}$ & $\begin{array}{r}71.0 \\
109.7 \\
186.1\end{array}$ & $\begin{array}{l}\text { Unit } 3 \text { : } \\
\text { Calcareous } \\
\text { silty clay and } \\
\text { clayey silt }\end{array}$ & $\begin{array}{l}\text { Quat.- } \\
\text { Plioc. }\end{array}$ & $\begin{array}{l}\text { Mica } \\
\text { Calc. } \\
\text { Mica. }\end{array}$ & $\begin{array}{l}\text { Quar. } \\
\text { Mica } \\
\text { Quar. }\end{array}$ & Quar. & $\begin{array}{l}\text { Mica } \\
\text { Quar. } \\
\text { Quar. }\end{array}$ & $\begin{array}{l}\text { Quar. } \\
\text { Mica. } \\
\text { Mica }\end{array}$ & $\begin{array}{l}\text { Plag. } \\
\text { Plag. } \\
\text { Plag. }\end{array}$ & $\begin{array}{l}\text { Mica } \\
\text { Mont. } \\
\text { Mica }\end{array}$ & $\begin{array}{l}\text { Quar. } \\
\text { Mica } \\
\text { Mont. }\end{array}$ & $\begin{array}{l}\text { Chlo. } \\
\text { Quar. } \\
\text { Quar. }\end{array}$ \\
\hline $\begin{array}{l}13-2,110-112 \\
15-1,95-98 \\
16-1,140-142\end{array}$ & $\begin{array}{l}376.6 \\
451.0 \\
460.9\end{array}$ & $\begin{array}{l}\text { Unit } 5 \text { : } \\
\text { Clayey silt } \\
\text { and silty clay }\end{array}$ & $\begin{array}{l}\text { Upper } \\
\text { Mioc. }\end{array}$ & $\begin{array}{l}\text { Mica } \\
\text { Mica } \\
\text { Mica }\end{array}$ & $\begin{array}{l}\text { Quar. } \\
\text { Quar. } \\
\text { Quar. }\end{array}$ & Calc. & $\begin{array}{l}\text { Quar. } \\
\text { Mica } \\
\text { Mica }\end{array}$ & $\begin{array}{l}\text { Mica } \\
\text { Quar. } \\
\text { Quar. }\end{array}$ & $\begin{array}{l}\text { Plag. } \\
\text { Plag. }\end{array}$ & $\begin{array}{l}\text { Mica } \\
\text { Mica } \\
\text { Mica }\end{array}$ & $\begin{array}{l}\text { Mont. } \\
\text { Quar. } \\
\text { Quar. }\end{array}$ & $\begin{array}{l}\text { Quar } \\
\text { Mont. } \\
\text { Mont. }\end{array}$ \\
\hline $\begin{array}{l}17-1,135-138 \\
19-1,136-140 \\
20-1,36-40 \\
21-1,88-92 \\
22-2,65-68\end{array}$ & $\begin{array}{l}470.4 \\
489.4 \\
497.9 \\
536.4 \\
575.7\end{array}$ & $\begin{array}{l}\text { Unit 6: } \\
\text { Interlaminated } \\
\text { silt, clayey } \\
\text { silt, and sandy } \\
\text { silt }\end{array}$ & $\begin{array}{l}\text { Upper } \\
\text { Mioc. }\end{array}$ & $\begin{array}{l}\text { Mica } \\
\text { Mica } \\
\text { Mica } \\
\text { Mica } \\
\text { Mica }\end{array}$ & $\begin{array}{l}\text { Quar. } \\
\text { Quar. } \\
\text { Quar. } \\
\text { Quar. } \\
\text { Quar. }\end{array}$ & & $\begin{array}{l}\text { Mica } \\
\text { Mica } \\
\text { Mica } \\
\text { Quar. } \\
\text { Mica }\end{array}$ & $\begin{array}{l}\text { Quar. } \\
\text { Quar. } \\
\text { Quar. } \\
\text { Mica } \\
\text { Quar. }\end{array}$ & $\begin{array}{l}\text { Plag. } \\
\text { Plag. } \\
\text { Plag. } \\
\text { Plag. }\end{array}$ & $\begin{array}{l}\text { Mica } \\
\text { Mica } \\
\text { Mica } \\
\text { Mica } \\
\text { Mica }\end{array}$ & $\begin{array}{l}\text { Quar. } \\
\text { Quar. } \\
\text { Quar. } \\
\text { Quar. } \\
\text { Quar. }\end{array}$ & $\begin{array}{l}\text { Chlo } \\
\text { Mont. } \\
\text { Mont. } \\
\text { Mont. } \\
\text { Mont. }\end{array}$ \\
\hline $\begin{array}{l}23-1,22-24 \\
23-2,78-80\end{array}$ & $\begin{array}{l}611.7 \\
613.8\end{array}$ & Unit $7^{a}$ & a & $\begin{array}{l}\text { Mica } \\
\text { Mont. }\end{array}$ & $\begin{array}{l}\text { Quar. } \\
\text { Mica }\end{array}$ & $\begin{array}{l}\text { Chlo. } \\
\text { Quar. }\end{array}$ & $\begin{array}{l}\text { Mica } \\
\text { Quar. }\end{array}$ & $\begin{array}{l}\text { Quar. } \\
\text { Mica }\end{array}$ & $\begin{array}{l}\text { Plag. } \\
\text { Plag }\end{array}$ & $\begin{array}{l}\text { Mica } \\
\text { Mont. }\end{array}$ & $\begin{array}{l}\text { Quar. } \\
\text { Kaol. }\end{array}$ & $\begin{array}{l}\text { Mont. } \\
\text { Mica. }\end{array}$ \\
\hline $\begin{array}{l}24-2,82-84 \\
25-2,95-97 \\
26-1,120-122 \\
27-1,130-132\end{array}$ & $\begin{array}{l}651.8 \\
690.0 \\
726.7 \\
764.8\end{array}$ & $\begin{array}{l}\text { Unit 8: } \\
\text { Interlaminated } \\
\text { silt, clayey silt } \\
\text { and sandy silt }\end{array}$ & $\begin{array}{l}\text { Middle } \\
\text { Mioc. }\end{array}$ & $\begin{array}{l}\text { Mica } \\
\text { Mica } \\
\text { Mica } \\
\text { Mica }\end{array}$ & $\begin{array}{l}\text { Quar. } \\
\text { Quar. } \\
\text { Quar. } \\
\text { Quar. }\end{array}$ & $\begin{array}{l}\text { Plag. } \\
\text { Plag. } \\
\text { Chlo. } \\
\text { Plag. }\end{array}$ & $\begin{array}{l}\text { Mica } \\
\text { Mica } \\
\text { Insuf }\end{array}$ & $\begin{array}{l}\text { Quar. } \\
\text { Quar. } \\
\text { cient re }\end{array}$ & $\begin{array}{l}\text { Plag. } \\
\text { Plag. } \\
\text { idue }\end{array}$ & $\begin{array}{l}\text { Mica } \\
\text { Mica }\end{array}$ & $\begin{array}{l}\text { Quar. } \\
\text { Mont. }\end{array}$ & $\begin{array}{l}\text { Mont. } \\
\text { Quar. }\end{array}$ \\
\hline
\end{tabular}

aUnit 7 is upper Miocene in age and consists of clayey silt and nanno ooze. 
TABLE 9

Results of X-Ray Diffraction Analysis from Hole 211

\begin{tabular}{|c|c|c|c|c|c|c|c|c|c|c|c|c|c|c|c|c|c|}
\hline Core & $\begin{array}{c}\text { Cored } \\
\text { Interval } \\
\text { Below Sea } \\
\text { Floor (m) }\end{array}$ & $\begin{array}{l}\text { Sample Deptha } \\
\text { Below Sea } \\
\text { Floor } \\
(\mathrm{m}) \\
\end{array}$ & 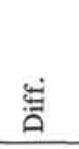 & $\begin{array}{l}\dot{:} \\
\text { 官 } \\
\text { ¿ }\end{array}$ & లું & $\stackrel{\circ}{\circ}$ & 己ే & 立 & $\frac{00}{20}$ & 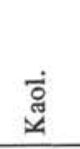 & 迅 & 宅 & 茫 & 烏 & 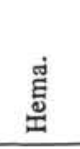 & نें & $\frac{\pi}{5}$ \\
\hline \multicolumn{18}{|c|}{ Bulk Samples } \\
\hline 1 & $0.0-9.0$ & 8.4 & 88.1 & 81.4 & - & - & 29.9 & 2.1 & 8.2 & 6.8 & 27.6 & 1.8 & 23.6 & - & - & - & - \\
\hline 3 & $57: 0-66.0$ & 57.9 & 86.0 & 78.2 & - & - & 23.8 & 3.9 & 11.3 & 5.2 & 27.5 & 2.8 & 25.6 & - & - & - & - \\
\hline 4 & $95.0-104.5$ & 97.2 & 81.2 & 70.7 & - & - & 26.4 & 4.7 & 9.9 & 2.6 & 37.7 & 2.1 & 16.5 & - & - & - & - \\
\hline 5 & $133.0-142.5$ & 141.2 & 88.6 & 82.2 & - & - & 22.7 & 4.4 & 10.0 & 12.8 & 9.7 & - & 40.5 & - & - & - & - \\
\hline 6 & $180.5-190.5$ & 189.1 & 91.1 & 86.1 & - & - & 12.9 & 4.4 & 11.9 & 12.0 & 8.6 & - & 50.2 & - & - & - & - \\
\hline 10 & $342.0-351.0$ & 351.0 & 81.6 & 71.3 & - & - & 16.5 & 8.2 & 2.1 & 20.9 & 8.5 & 1.4 & 42.4 & - & - & - & $\mathrm{P}$ \\
\hline 11 & $389.5-398.0$ & 398.0 & 89.5 & 83.6 & - & - & 13.0 & 35.6 & - & 1.8 & 12.8 & 1.5 & 14.7 & 16.9 & 3.7 & $\mathrm{P}$ & $\mathrm{T}$ \\
\hline \multirow[t]{3}{*}{12} & $409.0-418.5$ & 409.1 & 84.3 & 75.4 & 34.4 & - & 7.6 & 11.8 & - & 0.3 & 4.9 & 1.0 & 2.3 & 37.9 & - & - & $\mathrm{T}$ \\
\hline & & 411.8 & 71.4 & 55.3 & 34.9 & - & 14.9 & 29.2 & - & - & 14.1 & - & - & - & 6.9 & - & $\mathrm{T}$ \\
\hline & & 411.9 & 72.3 & 56.8 & 81.5 & - & 4.8 & 6.7 & - & - & 7.0 & - & - & - & - & - & $\mathrm{T}$ \\
\hline \multirow[t]{2}{*}{13} & $418.5-428.0$ & 418.6 & 87.9 & 81.1 & 32.6 & - & 9.5 & 15.5 & - & - & 4.0 & - & 10.2 & 28.2 & - & - & $\mathrm{P}$ \\
\hline & & 419.5 & 86.0 & 78.1 & 35.1 & - & 6.0 & 5.9 & - & - & 5.9 & - & - & 47.1 & - & - & $\mathrm{T}$ \\
\hline 14 & $428.0-437.5$ & 428.1 & 85.3 & 77.1 & 3.3 & 5.7 & 13.2 & 8.8 & - & - & - & - & - & 68.9 & - & - & - \\
\hline \multicolumn{18}{|c|}{ 2-20 $\mu$ m Fraction } \\
\hline 1 & $0.0-9.0$ & 8.4 & 81.7 & 71.4 & & & 50.3 & 5.2 & 19.7 & 1.9 & 19.6 & 3.3 & - & - & - & & \\
\hline 3 & $57.0-66.0$ & 57.9 & 82.6 & 72.8 & & & 42.7 & 6.7 & 19.9 & 2.0 & 25.9 & 2.9 & - & - & - & & \\
\hline 4 & $95.0-104.5$ & 97.2 & 76.9 & 64.0 & & & 42.9 & 8.4 & 18.6 & - & 26.2 & 4.0 & - & - & - & & \\
\hline 5 & $133.0-142.5$ & 141.2 & 87.1 & 79.9 & & & 24.9 & 5.7 & 16.3 & 10.4 & 9.2 & - & 33.4 & - & - & & \\
\hline 6 & $180.5-190.5$ & 189.1 & 91.2 & 86.2 & & & 18.0 & 7.7 & 22.1 & 11.2 & 6.0 & - & 34.9 & - & - & & \\
\hline 11 & $389.5-398.0$ & 398.0 & 78.6 & 66.5 & & & 20.3 & 59.9 & - & - & 12.8 & 1.4 & - & - & 5.7 & & \\
\hline \multirow[t]{3}{*}{12} & $409.0-418.5$ & 409.1 & 67.9 & 49.8 & & & 34.6 & 50.7 & - & 0.7 & 13.1 & 0.8 & - & - & - & & \\
\hline & & 411.8 & 62.5 & 41.3 & & & 29.2 & 56.8 & - & - & 10.7 & - & - & - & 3.3 & & \\
\hline & & 411.9 & 63.5 & 43.0 & & & 37.4 & 50.2 & - & - & 10.3 & 0.8 & - & - & 1.2 & & \\
\hline \multirow[t]{2}{*}{13} & $418.5-428.0$ & 418.6 & 84.5 & 75.7 & & & 35.3 & 45.6 & - & - & 9.6 & - & - & 9.5 & - & & \\
\hline & & 419.5 & 69.4 & 52.2 & & & 51.0 & 43.0 & - & - & 6.0 & - & - & - & - & & \\
\hline 14 & $428.0-437.5$ & 428.1 & 79.2 & 67.6 & & & 53.2 & 43.8 & - & - & 3.1 & - & - & - & - & & \\
\hline \multicolumn{18}{|c|}{$<2 \mu \mathrm{m}$ Fraction } \\
\hline 1 & $0.0-9.0$ & 8.4 & 82.6 & 72.8 & & & 13.9 & - & 2.7 & 17.8 & 20.7 & 1.6 & 43.4 & - & - & - & \\
\hline 3 & $57.0-66.0$ & 57.9 & 83.3 & 73.9 & & & 13.6 & 1.1 & 4.5 & 15.0 & 17.3 & 1.5 & 47.1 & - & - & - & \\
\hline 4 & $95.0-104.5$ & 97.2 & 77.9 & 65.5 & & & 6.0 & 1.0 & 1.8 & 2.3 & 5.3 & 1.7 & 81.9 & - & - & - & \\
\hline 5 & $133.0-142.5$ & 141.2 & 86.7 & 79.2 & & & 11.2 & - & 4.9 & 10.0 & 6.6 & - & 67.2 & - & - & - & \\
\hline 6 & $180.5-190.5$ & 189.1 & 85.8 & 77.7 & & & 7.9 & 2.9 & 4.3 & 7.7 & 4.1 & - & 73.1 & - & - & - & \\
\hline 10 & $342.0-351.0$ & 351.0 & 79.4 & 67.8 & & & 10.4 & 5.7 & 1.0 & 21.8 & 10.7 & - & 50.3 & - & - & - & \\
\hline 11 & $389.5-398.0$ & 398.0 & 89.3 & 83.2 & & & 11.0 & 26.4 & - & - & - & - & 43.5 & 14.5 & 4.6 & $P$ & \\
\hline \multirow[t]{3}{*}{12} & $409.0-418.5$ & 409.1 & 90.5 & 85.2 & & & 7.8 & 8.6 & - & 1.9 & 4.9 & 1.3 & 11.9 & 63.5 & - & - & \\
\hline & & 411.8 & 80.5 & 69.6 & & & 15.8 & 28.3 & - & - & 32.4 & - & 2.4 & - & 21.0 & - & \\
\hline & & 411.9 & 90.9 & 85.8 & & & 14.9 & 16.9 & - & - & 29.7 & 2.1 & 32.7 & - & 3.7 & - & \\
\hline \multirow[t]{2}{*}{13} & $418.5-428.0$ & 418.6 & 90.6 & 85.3 & & & 7.1 & 10.8 & - & 0.6 & 13.3 & 1.7 & 35.1 & 31.4 & - & - & \\
\hline & & 419.5 & 89.9 & 84.2 & & & 7.3 & 10.8 & - & 0.4 & 9.2 & 1.3 & 12.4 & 58.6 & - & - & \\
\hline 14 & $428.0-437.5$ & 428.1 & 82.5 & 72.7 & & & 14.0 & 16.3 & - & - & 4.4 & - & 2.4 & 62.8 & - & - & \\
\hline
\end{tabular}

aU-1 Peak at $6.0 \AA . . P=$ present; $t=$ trace. 
TABLE 10

Results of X-Ray Diffraction Analysis from Hole 212

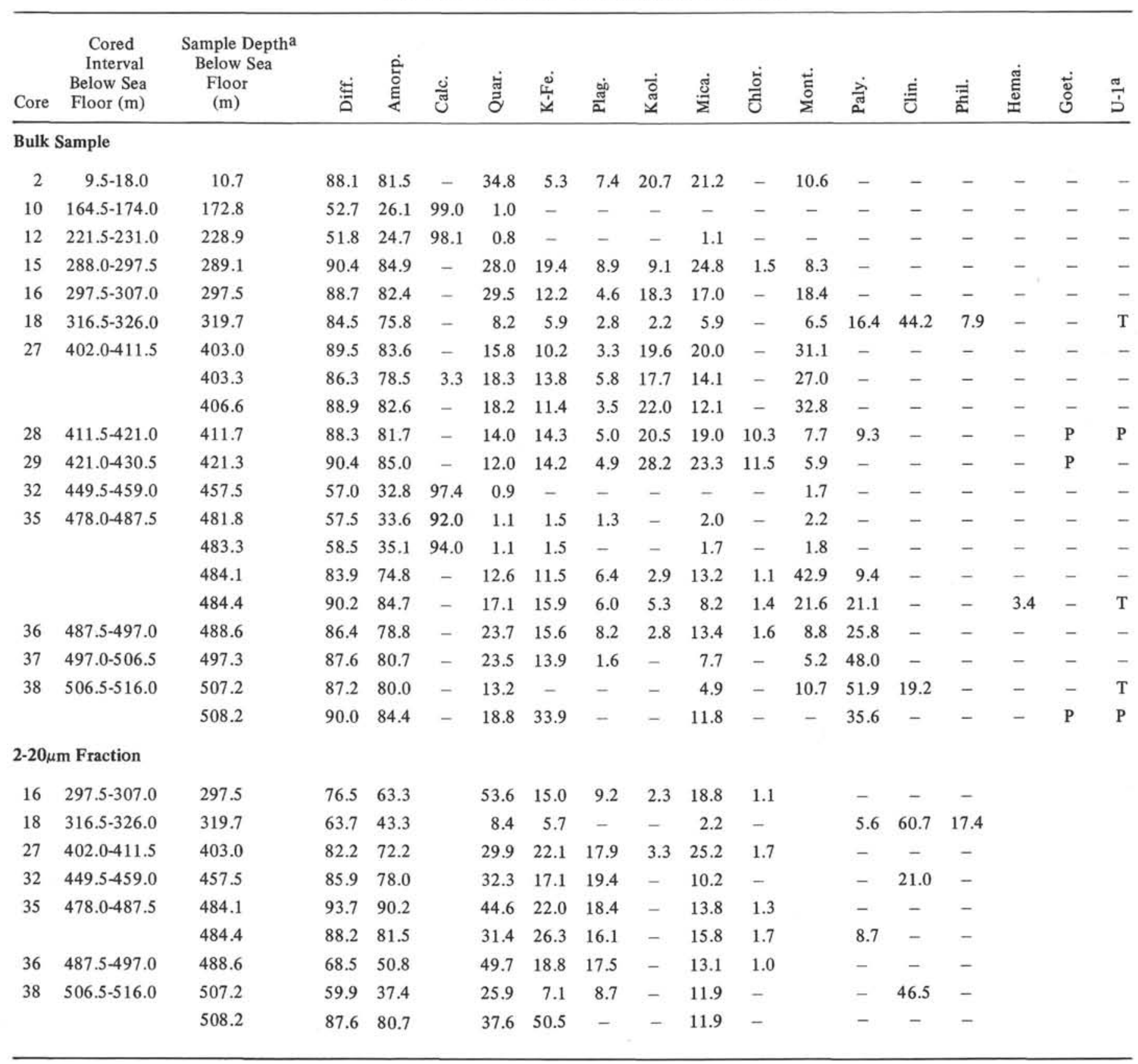


TABLE 10 - Continued

\begin{tabular}{|c|c|c|c|c|c|c|c|c|c|c|c|c|c|c|c|c|c|c|}
\hline Core & $\begin{array}{l}\text { Cored } \\
\text { Interval } \\
\text { Below Sea } \\
\text { Floor (m) }\end{array}$ & $\begin{array}{l}\text { Sample Deptha } \\
\text { Below Sea } \\
\text { Floor } \\
\text { (m) }\end{array}$ & 芯 & 客 & ల் & ڤँّ & 㭊 & $\frac{\text { å }}{\text { a }}$ & $\begin{array}{l}\dot{0} \\
\stackrel{\tilde{g}}{ }\end{array}$ & $\underset{\check{z}}{\stackrel{0}{\Sigma}}$ & 远 & $\stackrel{+}{\stackrel{\Xi}{\Sigma}}$ & 底 & 离 & 竎 & 冚 & 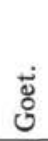 & $\frac{\pi}{d}$ \\
\hline \multicolumn{19}{|c|}{$<2 \mu \mathrm{m}$ Fraction } \\
\hline 2 & $9.5-18.0$ & 10.7 & 88.0 & 81.2 & & 18.7 & 7.0 & 5.0 & 27.1 & 11.4 & - & 30.8 & - & - & & & - & \\
\hline 10 & $164.5-174.0$ & 172.8 & 82.8 & 73.2 & & 7.6 & 3.5 & 1.9 & 13.5 & 12.9 & - & 54.3 & 6.3 & - & & & - & \\
\hline 12 & $221.5-231.0$ & 228.9 & 83.3 & 73.9 & & 8.9 & 4.8 & 2.2 & 15.6 & 10.1 & - & 58.4 & - & - & & & - & \\
\hline 15 & $288.0-297.5$ & 289.1 & 87.7 & 80.8 & & 13.8 & 8.3 & 3.4 & 13.0 & 10.3 & - & 33.6 & 17.6 & - & & & - & \\
\hline 16 & $297.5-307.0$ & 297.5 & 86.9 & 79.5 & & 19.6 & 6.5 & 1.7 & 21.8 & 14.6 & - & 35.8 & - & - & & & - & \\
\hline 18 & $316.5-326.0$ & 319.7 & 89.6 & 83.7 & & 8.8 & 12.2 & - & - & 10.5 & - & 39.7 & 21.5 & 7.3 & & & - & \\
\hline \multirow[t]{3}{*}{27} & $402.0-411.5$ & 403.0 & 85.5 & 77.3 & & 9.0 & 3.9 & 3.1 & 14.0 & 7.9 & 2.4 & 59.8 & - & - & & & - & \\
\hline & & 403.3 & 80.3 & 69.2 & & 9.7 & 4.9 & 2.1 & 16.6 & 9.5 & 2.1 & 55.0 & - & - & & & - & \\
\hline & & 406.6 & 86.5 & 78.9 & & 12.1 & 4.8 & 1.9 & 15.1 & 8.0 & - & 58.1 & - & - & & & - & \\
\hline 28 & $411.5-421.0$ & 411.7 & 84.3 & 75.4 & & 10.3 & 4.3 & - & 24.1 & 11.6 & 6.4 & 36.9 & 6.5 & - & & & $\mathbf{P}$ & \\
\hline 29 & $421.0-430.5$ & 421.3 & 88.0 & 81.3 & & 8.9 & 7.1 & 1.2 & 29.4 & 12.4 & 6.1 & 34.9 & - & - & & & - & \\
\hline 32 & $449.5-459.0$ & 457.5 & 81.1 & 70.5 & & 4.8 & 3.8 & 1.9 & 1.1 & 4.8 & - & 81.9 & - & 2.3 & & & - & \\
\hline \multirow[t]{4}{*}{35} & $478.0-487.5$ & 481.8 & 81.4 & 71.0 & & 6.1 & 4.5 & 1.4 & - & 5.0 & - & 83.0 & - & - & & & - & \\
\hline & & 483.3 & 79.5 & 68.0 & & 5.3 & 2.8 & 1.3 & - & 5.0 & - & 78.9 & 6.8 & - & & & - & \\
\hline & & 484.1 & 86.3 & 78.6 & & 12.4 & 7.4 & 1.9 & 1.8 & 7.1 & 2.3 & 45.8 & 21.3 & - & & & - & \\
\hline & & 484.4 & 86.6 & 79.0 & & 14.7 & 11.7 & - & - & 3.9 & 2.2 & 47.4 & 20.1 & - & & & - & \\
\hline 36 & $487.5-497.0$ & 488.6 & 90.2 & 84.8 & & 21.3 & 16.1 & - & 7.8 & 15.3 & 2.0 & - & 37.5 & - & & & - & \\
\hline 37 & $497.0-506.5$ & 497.3 & 88.9 & 82.7 & & 14.9 & 10.8 & - & 1.3 & 8.5 & 1.3 & 11.2 & 52.1 & - & & & - & \\
\hline \multirow[t]{2}{*}{38} & $506.5-516.0$ & 507.2 & 87.4 & 80.4 & & 9.3 & 8.5 & 1.1 & - & 11.3 & - & - & 52.6 & 17.3 & & & - & \\
\hline & & 508.2 & 88.0 & 81.2 & & 10.1 & 18.9 & - & - & 7.5 & - & 36.8 & 26.7 & - & & & $P$ & \\
\hline
\end{tabular}

aU-1 Peak at $6.00 \AA . T=$ trace; $\mathrm{P}=$ present. 
TABLE 11

Results of X-Ray Diffraction Analysis from Hole 213

\begin{tabular}{|c|c|c|c|c|c|c|c|c|c|c|c|c|c|c|c|c|c|}
\hline Core & $\begin{array}{c}\text { Cored } \\
\text { Interval } \\
\text { Below Sea } \\
\text { Floor (m) }\end{array}$ & $\begin{array}{l}\text { Sample Deptha } \\
\text { Below Sea } \\
\text { Floor } \\
\text { (m) }\end{array}$ & 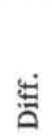 & $\begin{array}{l}\dot{2} \\
\dot{0} \\
\dot{\Xi}\end{array}$ & లే & $\ddot{\tilde{z}}$ & 荘 & $\stackrel{\dot{0}}{\frac{0}{\alpha}}$ & $\begin{array}{l}\text { gु } \\
\stackrel{g}{ }\end{array}$ & $\stackrel{\text { }}{\stackrel{\Xi}{\Sigma}}$ & 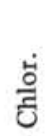 & 范 & 㐫 & 竎 & 家 & 过 & 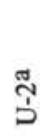 \\
\hline
\end{tabular}

Bulk Sample

\begin{tabular}{|c|c|c|c|c|c|c|c|c|c|c|c|c|c|c|c|c|c|}
\hline \multirow[t]{3}{*}{1} & $0.0-9.0$ & 0.1 & 93.3 & 89.6 & - & 27.9 & 4.5 & 12.1 & 15.2 & 23.7 & - & 16.7 & - & - & - & - & - \\
\hline & & 0.7 & 92.6 & 88.4 & - & 31.3 & - & 13.6 & 18.6 & 25.0 & - & 11.4 & - & - & - & - & - \\
\hline & & 5.8 & 93.9 & 90.5 & - & 35.7 & - & 10.8 & 21.4 & 23.6 & - & 8.4 & - & - & - & - & - \\
\hline \multirow[t]{2}{*}{2} & $9.0-18.5$ & 15.3 & 92.3 & 87.9 & - & 31.0 & 3.8 & 15.0 & 16.3 & 22.7 & - & 11.1 & - & - & - & - & - \\
\hline & & 15.5 & 91.7 & 87.0 & - & 34.8 & - & 11.7 & 18.2 & 22.6 & - & 12.7 & - & - & - & - & - \\
\hline 3 & $18.5-28.0$ & 26.1 & 92.4 & 88.1 & - & 26.1 & - & 7.9 & 19.4 & 29.7 & - & 17.0 & - & - & - & - & - \\
\hline 6 & $47.0-56.5$ & 53.7 & 91.6 & 86.9 & - & 26.3 & 3.4 & 11.4 & 17.8 & 25.1 & - & 15.9 & - & - & - & - & - \\
\hline \multirow[t]{2}{*}{8} & $66.0-75.5$ & 69.1 & 91.2 & 86.3 & - & 28.9 & - & 10.4 & 19.0 & 16.1 & - & 25.6 & - & - & - & - & - \\
\hline & & 74.9 & 86.9 & 79.6 & - & 15.2 & 3.2 & 7.1 & 10.6 & 13.9 & - & 50.1 & - & - & - & - & - \\
\hline 9 & $75.5-85.0$ & 83.8 & 89.2 & 83.1 & - & 12.8 & 10.6 & 6.5 & 11.2 & 12.6 & - & 36.3 & - & 10.2 & - & - & - \\
\hline 10 & $85.0-94.5$ & 88.7 & 89.4 & 83.4 & - & 14.5 & - & 9.1 & 14.5 & 9.1 & - & 37.4 & - & 15.5 & - & - & - \\
\hline 11 & $94.5-104.0$ & 99.7 & 89.5 & 83.7 & - & 9.4 & 4.7 & 6.3 & 4.2 & 6.7 & - & 14.9 & 14.3 & 38.1 & 1.4 & - & - \\
\hline 13 & $113.5-123.0$ & 117.2 & 88.2 & 81.6 & - & 10.3 & 10.3 & - & - & 7.0 & - & 5.4 & 19.8 & 47.1 & - & - & - \\
\hline 14 & $123.0-132.5$ & 125.2 & 89.8 & 84.1 & - & 12.9 & 24.6 & - & 0.9 & 13.5 & 2.4 & 10.5 & 35.3 & - & - & - & - \\
\hline \multirow[t]{2}{*}{16} & $142.0-151.5$ & 144.6 & 44.8 & 13.7 & 100.0 & - & - & - & - & - & - & - & - & - & - & - & $\mathrm{T}$ \\
\hline & & 147.9 & 91.3 & 86.3 & 12.5 & 11.0 & 15.5 & - & 3.9 & - & - & 2.6 & 54.5 & - & - & $\mathbf{P}$ & - \\
\hline
\end{tabular}

2-20 $\mu \mathrm{m}$ Fraction

\begin{tabular}{|c|c|c|c|c|c|c|c|c|c|c|c|c|c|}
\hline $85.0-94.5$ & 88.7 & 95.6 & 93.1 & 30.3 & - & 4.2 & - & - & - & 24.5 & - & 41.1 & - \\
\hline $94.5-104.0$ & 99.7 & 71.4 & 55.4 & 6.4 & 7.1 & - & - & 2.9 & - & - & - & 83.6 & - \\
\hline $113.5-123.0$ & 117.2 & 73.5 & 58.5 & 8.6 & 5.9 & - & - & 7.2 & - & - & 8.0 & 70.2 & - \\
\hline $123.0-132.5$ & 125.2 & 85.1 & 76.7 & 28.7 & 31.6 & - & - & 10.9 & 1.5 & - & 27.4 & - & - \\
\hline $142.0-151.5$ & 144.6 & 85.1 & 76.8 & 57.6 & 42.4 & - & - & - & - & - & - & - & - \\
\hline & 147.9 & 85.7 & 77.6 & 22.1 & 43.8 & - & 1.7 & 17.8 & 1.4 & - & 13.1 & - & $\mathbf{P}$ \\
\hline
\end{tabular}

$<2 \mu \mathrm{m}$ Fraction

\begin{tabular}{|c|c|c|c|c|c|c|c|c|c|c|c|c|c|c|c|}
\hline \multirow[t]{3}{*}{1} & $0.0-9.0$ & 0.1 & 90.0 & 84.4 & 19.1 & 7.1 & 7.3 & 16.4 & 16.5 & - & 33.6 & - & - & - & - \\
\hline & & 0.7 & 91.1 & 86.2 & 22.0 & - & 8.1 & 17.7 & 19.2 & - & 33.0 & - & - & - & - \\
\hline & & 5.8 & 92.0 & 87.6 & 27.3 & - & 5.6 & 26.4 & 14.8 & - & 25.9 & - & - & - & - \\
\hline \multirow[t]{2}{*}{2} & $9.0-18.5$ & 15.3 & 88.3 & 81.8 & 18.6 & 3.7 & 5.9 & 19.9 & 16.8 & - & 35.1 & - & - & - & - \\
\hline & & 15.5 & 89.6 & 83.7 & 26.9 & - & 9.9 & 20.2 & 14.3 & - & 28.7 & - & - & - & - \\
\hline 3 & $18.5-28.0$ & 26.1 & 88.7 & 82.3 & 11.8 & 4.0 & 3.7 & 19.0 & 12.4 & - & 49.2 & - & - & - & - \\
\hline 6 & $47.0-56.5$ & 53.7 & 88.1 & 81.4 & 14.9 & 5.0 & 4.0 & 22.4 & 13.9 & - & 39.7 & - & - & - & - \\
\hline \multirow[t]{2}{*}{8} & $66.0-75.5$ & 69.1 & 86.4 & 78.7 & 13.5 & - & 6.0 & 19.0 & 7.8 & - & 53.6 & - & - & - & - \\
\hline & & 74.9 & 84.1 & 75.2 & 12.0 & 5.0 & 3.6 & 11.6 & - & - & 67.9 & - & - & - & - \\
\hline 9 & $75.5-85.0$ & 83.8 & 83.8 & 74.7 & 6.5 & 4.1 & 1.4 & 10.2 & 3.7 & - & 64.8 & 5.7 & 3.5 & - & - \\
\hline 10 & $85.0-94.5$ & 88.7 & 83.3 & 73.9 & 9.3 & - & 4.9 & 8.6 & 3.8 & - & 73.4 & - & - & - & - \\
\hline 11 & $94.5-104.0$ & 99.7 & 88.0 & 81.3 & 12.5 & 9.9 & - & 12.2 & 8.1 & - & 41.3 & 12.5 & 3.5 & - & - \\
\hline 13 & $113.5-123.0$ & 117.2 & 86.3 & 78.6 & 12.0 & 11.7 & - & - & 10.5 & - & 18.1 & 11.4 & 36.4 & - & - \\
\hline 14 & $123.0-132.5$ & 125.2 & 90.7 & 85.4 & 9.1 & 12.5 & - & 1.4 & 7.1 & 2.1 & 38.1 & 29.6 & - & - & - \\
\hline \multirow[t]{2}{*}{16} & $142.0-151.5$ & 144.6 & 57.0 & 32.8 & - & - & - & - & - & - & - & - & - & - & M \\
\hline & & 147.9 & 91.8 & 87.1 & 7.7 & 17.0 & - & 6.0 & - & - & 12.0 & 57.3 & - & $\mathrm{P}$ & - \\
\hline
\end{tabular}

aU-2 Peaks at $3.02 \AA, 7.81 \AA, 3.96 \AA, 5.33 \AA, 2.67 \AA$, and about 50 others. $\mathrm{M}=$ major; $\mathrm{P}=$ present; $\mathrm{T}=$ trace. 
TABLE 12

Results of X-Ray Diffraction Analysis from Hole 214

\begin{tabular}{|c|c|c|c|c|c|c|c|c|c|c|c|c|c|c|c|c|c|c|c|c|c|}
\hline Core & $\begin{array}{c}\text { Cored } \\
\text { Interval } \\
\text { Below Sea } \\
\text { Floor }(\mathrm{m})\end{array}$ & $\begin{array}{l}\text { Sample Deptha } \\
\text { Below Sea } \\
\text { Floor } \\
\text { (m) }\end{array}$ & 岕 & $\begin{array}{l}\dot{0} \\
\text { : } \\
\text { 定 }\end{array}$ & गु & 爮 & 荀 & ฮี & 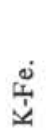 & $\stackrel{\Xi}{20}$ & ت्ञ & 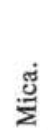 & $\stackrel{0}{\Sigma}$ & 竎 & 院 & 范 & 苂 & 莺 & 仓் & 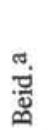 & $\stackrel{\text { p }}{\dot{s}}$ \\
\hline
\end{tabular}

\section{Bulk Sample}

$3 \quad 19.0-28$

$36 \quad 333.0-342.5 \quad 23.4$

334.6

336.5

$37 \quad 342.5-352.0 \quad 345.2$

$38 \quad 352.0-361.5 \quad 356.5$

$40 \quad 371.0-380.5 \quad 372.1$

$41 \quad 380.5-390.0 \quad 384.5$

384.8

$42 \quad 390.0-399.5 \quad 390.9$

391.1

$44 \quad 402.5-412.0 \quad 403.4$

403.6

$46 \quad 421.5-431.0 \quad 423.3$

424.4

$\begin{array}{lll}52 & 476.5-486.0 \quad 477.8\end{array}$

\section{2-20 $\mu$ m Fraction}

$\begin{array}{lllll}36 & 333.0-342.5 & 334.6 & 94.7 & 91.7 \\ & & 336.5 & 58.5 & 35.1 \\ 37 & 342.5-352.0 & 345.2 & 88.3 & 81.7 \\ 38 & 352.0-361.5 & 356.5 & 84.4 & 75.6 \\ 40 & 371.0-380.5 & 372.1 & 79.1 & 67.3 \\ 41 & 380.5-390.0 & 384.5 & 61.8 & 40.3 \\ & & 384.8 & 56.0 & 31.2 \\ 42 & 390.0-399.5 & 390.9 & 92.4 & 88.2 \\ & & 391.1 & 63.8 & 43.5 \\ 44 & 402.5-412.0 & 403.4 & 79.6 & 68.1 \\ & & 403.6 & 74.1 & 59.5 \\ 46 & 421.5-431.0 & 423.3 & 63.9 & 43.6 \\ & & 424.4 & 83.6 & 74.4 \\ 52 & 476.5-486.0 & 477.8 & 88.9 & 82.7\end{array}$

$\begin{array}{lllllllllllllllllll}50.1 & 22.0 & 100.0 & - & - & - & - & - & - & - & - & - & - & - & - & - & - & - \\ 49.3 & 20.8 & 100.0 & - & - & - & - & - & - & - & - & - & - & - & - & - & - & - \\ 78.6 & 66.5 & - & - & - & - & - & 40.5 & - & - & 38.6 & - & - & - & 20.9 & - & \mathrm{T} & - \\ 76.9 & 63.9 & 55.4 & - & - & - & - & 12.3 & - & 3.9 & 6.4 & 21.9 & - & - & - & - & \mathrm{T} & - \\ 68.9 & 51.5 & 87.8 & - & - & - & - & 8.0 & - & - & 2.9 & - & - & 1.3 & - & - & - & - \\ 75.2 & 61.3 & 76.5 & - & - & - & - & 12.8 & - & - & 8.7 & - & - & 1.9 & - & - & - & - \\ 81.7 & 71.5 & 26.2 & 12.0 & - & 1.1 & - & 39.7 & - & - & 15.2 & - & - & 5.7 & - & - & - & - \\ 72.4 & 56.8 & 12.1 & - & - & 0.7 & - & 38.9 & - & - & 21.4 & - & - & 26.8 & - & - & - & - \\ 70.8 & 54.4 & 7.1 & - & - & - & - & 30.6 & - & - & 38.5 & - & - & 23.9 & - & - & - & - \\ 88.3 & 81.6 & - & - & - & - & - & - & - & - & - & 100.0 & - & - & - & - & - & \mathrm{M} \\ 83.6 & 74.3 & - & - & - & 2.5 & - & - & - & - & - & 91.1 & 1.9 & 4.5 & - & - & - & \mathrm{P} \\ 85.4 & 77.2 & - & - & - & - & 17.1 & 4.6 & 3.7 & - & 56.1 & - & - & 14.5 & - & 3.9 & - & \mathrm{A} \\ 88.4 & 81.9 & - & - & - & 12.9 & 59.6 & - & 20.6 & - & - & - & - & 6.9 & - & - & - & \mathrm{A} \\ 76.4 & 63.1 & - & - & - & 10.2 & 53.5 & - & - & - & 33.9 & - & - & 2.4 & - & - & - & \mathrm{P} \\ 86.0 & 78.1 & - & - & - & 3.8 & 15.7 & - & - & - & 65.1 & - & - & 15.5 & - & - & - & \mathrm{P} \\ 90.6 & 85.3 & 6.3 & - & 5.4 & 2.7 & 23.0 & - & - & - & 62.6 & - & - & - & - & - & - & \mathrm{P}\end{array}$

$\begin{array}{lcccc}- & 2.6 & - & 74.2 & - \\ - & - & - & 21.9 & - \\ - & 2.2 & - & 69.0 & - \\ - & - & - & 42.0 & - \\ - & 05 & - & 44.7 & - \\ - & 0.3 & - & 28.7 & - \\ - & - & - & 31.3 & - \\ - & 10.5 & 24.0 & - & - \\ - & 1.7 & - & - & - \\ - & 2.3 & 35.0 & 7.4 & 1.7 \\ - & 16.9 & 73.6 & - & - \\ - & 14.4 & 82.6 & - & - \\ 4.8 & 10.2 & 42.7 & - & - \\ 9.0 & 5.0 & 58.1 & - & -\end{array}$

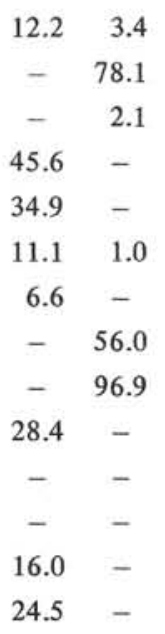




\section{$<2 \mu$ m Fraction}

\begin{tabular}{|c|c|c|c|c|c|c|c|c|c|c|c|c|c|c|c|c|c|}
\hline \multirow[t]{2}{*}{3} & $19.0-28.5$ & 19.3 & 96.0 & 93.8 & 19.3 & - & 7.7 & 18.4 & 13.2 & 41.4 & - & - & - & - & - & - & - \\
\hline & & 23.4 & 98.1 & 97.0 & 30.5 & - & 8.0 & 25.2 & 29.6 & 6.7 & - & - & - & - & - & - & - \\
\hline \multirow[t]{2}{*}{36} & $333.0-342.5$ & 334.6 & 92.5 & 88.2 & - & - & 27.7 & - & - & 50.3 & 2.0 & - & 20.0 & - & $P$ & - & - \\
\hline & & 336.5 & 91.9 & 87.4 & - & - & 37.6 & - & - & 51.9 & 3.9 & - & 6.5 & - & $\mathrm{P}$ & - & - \\
\hline 37 & $342.5-352.0$ & 345.2 & 84.4 & 75.6 & - & - & 17.1 & - & - & 77.7 & - & 5.2 & - & - & - & - & - \\
\hline 38 & $352.0-361.5$ & 356.5 & 88.4 & 81.8 & - & - & 8.7 & - & - & 82.0 & - & 9.3 & - & - & - & - & - \\
\hline 40 & $371.0-380.5$ & 372.1 & 86.0 & 78.1 & - & - & 11.7 & - & - & 84.3 & - & 4.1 & - & - & - & - & - \\
\hline \multirow[t]{2}{*}{41} & $380.5-390.0$ & 384.5 & 80.7 & 69.9 & - & - & 2.3 & - & - & 92.7 & - & 5.0 & - & - & - & - & - \\
\hline & & 384.8 & 78.0 & 65.7 & - & - & 4.1 & - & - & 91.8 & - & 4.1 & - & - & - & - & - \\
\hline \multirow[t]{2}{*}{42} & $390.0-399.5$ & 390.9 & 89.5 & 83.6 & - & - & - & - & - & - & - & - & - & - & - & M & - \\
\hline & & 391.1 & 93.7 & 90.1 & - & - & - & - & - & - & 81.7 & 18.3 & - & - & - & M & - \\
\hline \multirow[t]{2}{*}{44} & $402.5-412.0$ & 403.4 & 83.8 & 74.7 & - & - & - & 4.5 & - & 93.2 & - & - & - & 2.3 & - & $\mathbf{P}$ & - \\
\hline & & 403.6 & 91.7 & 87.0 & 6.7 & 15.3 & - & 20.1 & 48.2 & - & - & 9.7 & - & - & - & A & - \\
\hline \multirow[t]{2}{*}{46} & $421.5-431.0$ & 423.3 & 76.5 & 63.3 & 4.8 & 18.4 & - & - & - & 76.8 & - & - & - & - & - & - & A \\
\hline & & 424.4 & 85.4 & 77.3 & 1.4 & 5.4 & - & 3.0 & - & 87.9 & - & 2.3 & - & - & - & $P$ & - \\
\hline 52 & $476.5-486.0$ & 477.8 & 89.7 & 83.8 & - & - & - & - & 10.5 & 89.5 & - & - & - & - & - & $\mathrm{P}$ & - \\
\hline
\end{tabular}

Note: $\mathrm{T}=$ trace $\mathrm{M}=$ major $; \mathrm{P}$ - present $\mathrm{A}=$ abundant.

aBeid $=$ Beidellite.

bU-3 Peaks at $4.42 \AA$ (very narrow), $4.65 \AA$ (narrow), $3.80 \AA$ (broad), and others. 
TABLE 13

Results of X-Ray Diffraction Analysis from Hole 215

\begin{tabular}{|c|c|c|c|c|c|c|c|c|c|c|c|c|c|c|c|c|}
\hline Core & $\begin{array}{c}\text { Cored } \\
\text { Interval } \\
\text { Below Sea } \\
\text { Floor (m) }\end{array}$ & $\begin{array}{l}\text { Sample Deptha } \\
\text { Below Sea } \\
\text { Floor } \\
\text { (m) }\end{array}$ & 哭 & 官 & गु & ठีّ & 这 & $\frac{00}{20}$ & $\begin{array}{l}\dot{\vec{g}} \\
\stackrel{\Xi}{ }\end{array}$ & $\stackrel{\text { 릴 }}{\Sigma}$ & 客 & $\frac{\dot{E}}{\stackrel{E}{\Sigma}}$ & 永 & छ் & 竎 & 光 \\
\hline \multicolumn{17}{|c|}{ Bulk Sample } \\
\hline 1 & $0.0-9.5$ & 6.4 & 92.8 & 88.8 & - & 23.9 & - & 9.1 & 16.7 & 35.0 & - & 15.3 & - & & - & - \\
\hline 4 & $26.5-36.0$ & 27.0 & 93.8 & 90.3 & - & 26.7 & - & 5.9 & 19.4 & 23.6 & - & 24.4 & - & & - & - \\
\hline 6 & $45.5-55.0$ & 47.8 & 71.2 & 55.1 & - & 20.4 & 1.9 & 3.9 & - & 60.7 & 6.3 & 6.7 & - & & - & - \\
\hline 7 & $55.0-64.5$ & 57.1 & 92.8 & 88.8 & - & 28.0 & - & 7.8 & 15.9 & 28.2 & - & 20.1 & - & & - & - \\
\hline \multirow[t]{2}{*}{8} & $64.5-74.0$ & 66.6 & 84.3 & 75.4 & - & 18.8 & - & 8.7 & 6.9 & 15.2 & - & 50.5 & - & & - & - \\
\hline & & 69.2 & 75.9 & 62.3 & - & 25.4 & 2.8 & 6.1 & 1.1 & 51.9 & 5.5 & 7.3 & - & & - & - \\
\hline \multirow[t]{3}{*}{9} & $74.0-83.5$ & 74.4 & 88.1 & 81.4 & - & 9.8 & 7.6 & - & - & 6.4 & - & 12.7 & 36.1 & & 27.3 & - \\
\hline & & 76.1 & 85.6 & 77.5 & - & 8.5 & - & - & - & - & - & - & 91.5 & & - & - \\
\hline & & 77.4 & 81.6 & 71.3 & - & 6.8 & - & - & - & - & - & - & 88.6 & & - & 4.6 \\
\hline 16 & $140.5-150.0$ & 147.8 & 52.6 & 25.9 & 100.0 & - & - & - & - & - & - & - & - & & - & - \\
\hline \multicolumn{17}{|c|}{ 2-20 $\mu \mathrm{m}$ Fraction } \\
\hline 7 & $55.0-64.5$ & 57.1 & 91.0 & 85.9 & & 54.2 & - & 20.5 & 7.2 & 18.1 & - & - & - & - & - & \\
\hline \multirow[t]{2}{*}{8} & $64.5-74.0$ & 66.6 & 82.7 & 72.9 & & 29.9 & 2.9 & 12.4 & 5.4 & 26.2 & - & 23.2 & - & - & - & \\
\hline & & 69.2 & 64.9 & 45.2 & & 32.6 & 3.0 & 9.3 & - & 49.2 & 5.9 & - & - & - & - & \\
\hline \multirow[t]{2}{*}{9} & $74.0-83.5$ & 74.4 & 80.5 & 69.6 & & 15.9 & 13.7 & 6.1 & - & 8.8 & - & - & 7.0 & 1.2 & 47.3 & \\
\hline & & 77.4 & 97.9 & 96.7 & & 54.2 & 17.5 & 13.8 & - & - & - & - & 14.4 & - & - & \\
\hline \multicolumn{17}{|c|}{$<2 \mu \mathrm{m}$ Fraction } \\
\hline 1 & $0.0-9.5$ & 6.4 & 92.9 & 88.8 & & 14.1 & - & 3.5 & 12.9 & 35.4 & - & 34.1 & - & & - & - \\
\hline 4 & $26.5-36.0$ & 27.0 & 90.3 & 84.8 & & 14.2 & - & 3.7 & 20.6 & 12.6 & - & 48.9 & - & & - & - \\
\hline 6 & $45.5-55.0$ & 47.8 & 76.6 & 63.5 & & 17.4 & - & 1.7 & 5.4 & 42.4 & 5.8 & 27.3 & - & & - & - \\
\hline 7 & $55.0-64.5$ & 57.1 & 88.0 & 81.3 & & 15.7 & - & 4.6 & 15.6 & 11.3 & - & 52.8 & - & & - & - \\
\hline \multirow[t]{2}{*}{8} & $64.5-74.0$ & 66.6 & 83.5 & 74.2 & & 9.1 & - & 2.1 & 6.0 & 5.7 & - & 77.2 & - & & - & - \\
\hline & & 69.2 & 80.8 & 69.9 & & 16.8 & 1.2 & 3.3 & 8.2 & 38.6 & 2.8 & 29.0 & - & & - & - \\
\hline \multirow[t]{3}{*}{9} & $74.0-83.5$ & 74.4 & 88.2 & 81.5 & & 5.1 & - & - & 2.2 & - & - & 43.9 & 44.1 & & 4.7 & - \\
\hline & & 76.1 & 89.3 & 83.3 & & 4.3 & - & - & - & - & - & 5.7 & 88.3 & & - & 1.7 \\
\hline & & 77.4 & 87.6 & 80.6 & & 6.1 & - & - & - & - & - & 2.9 & 87.5 & & - & 3.6 \\
\hline 16 & $140.5-150.0$ & 147.8 & 92.2 & 87.8 & & - & 9.6 & - & 6.4 & - & - & 84.0 & - & & - & - \\
\hline
\end{tabular}


TABLE 14

Results of X-Ray Diffraction Analy sis from Hole 216

\begin{tabular}{|c|c|c|c|c|c|c|c|c|c|c|c|c|c|c|c|c|c|c|c|c|c|c|c|c|}
\hline Core & $\begin{array}{l}\text { Cored } \\
\text { Interval } \\
\text { Below Sea } \\
\text { Floor }(\mathrm{m})\end{array}$ & $\begin{array}{l}\text { Sample Deptha } \\
\text { Below Sea } \\
\text { Floor } \\
\text { (m) }\end{array}$ & $\overleftrightarrow{\mathscr{a}}$ & $\begin{array}{l}\dot{2} \\
\dot{0} \\
\dot{\varepsilon}\end{array}$ & $\frac{\dot{U}}{\tilde{U}}$ & $\dot{\tilde{z}}$ & 感 & 递 & 远 & 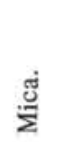 & 追 & $\frac{\vec{E}}{\stackrel{\Xi}{\Sigma}}$ & त्रें & 苞 & 㝘 & 夰 & 忘 & 홈 & 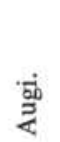 & 迅 & $\begin{array}{l}\text { 品. } \\
\text { ङ }\end{array}$ & $\stackrel{0}{7}$ & is & $\begin{array}{l}\dot{\varphi} \\
\dot{\rho}\end{array}$ \\
\hline
\end{tabular}

\section{Bulk Samples}

$\begin{array}{rrr}4 & 120.5-130.0 & 121.1 \\ 19 & 291.5-301.0 & 292.6 \\ 21 & 310.5-320.0 & 311.2 \\ 24 & 339.0-348.5 & 345.9 \\ 25 & 348.5-358.0 & 353.9 \\ 26 & 358.0-367.5 & 360.2 \\ 30 & 396.0-405.5 & 396.9 \\ 31 & 405.5-415.0 & 406.6 \\ 32 & 415.0-424.5 & 418.0 \\ 33 & 424.5-434.0 & 425.5 \\ 34 & 434.0-443.5 & 434.5 \\ & & 439.2 \\ & & 439.2\end{array}$

$\begin{array}{cccl}51.3 & 23.9 & 100.0 & - \\ 50.5 & 22.6 & 87.5 & 0.5 \\ 44.5 & 13.4 & 99.4 & 0.6 \\ 63.6 & 43.1 & 94.6 & 0.5 \\ 77.3 & 64.6 & 47.8 & - \\ 76.5 & 63.3 & 27.1 & 0.4 \\ 82.7 & 72.9 & 69.4 & 0.8 \\ 82.3 & 72.3 & 71.7 & - \\ 76.0 & 62.4 & 87.2 & 0.6 \\ 79.9 & 68.5 & 14.8 & - \\ 71.8 & 56.0 & 36.9 & - \\ 70.4 & 53.7 & 48.7 & 0.7 \\ 87.5 & 80.5 & - & -\end{array}$

\section{2-20 $\mu$ m Fraction}

$\begin{array}{lll}24 & 339.0-348.5 & 345.9 \\ 25 & 348.5-358.0 & 353.9 \\ 26 & 358.0-367.5 & 360.2 \\ 30 & 396.0-405.5 & 396.9 \\ 31 & 405.5-415.0 & 406.6 \\ 32 & 415.0-424.5 & 418.0 \\ 33 & 424.5-434.0 & 425.5 \\ 34 & 434.0-443.5 & 434.5 \\ & & 439.2 \\ & & 439.2\end{array}$

$\begin{array}{rrllr}81.3 & 70.7 & 7.6 & 5.7 & 6.4 \\ 78.7 & 66.7 & 1.2 & - & 24.5 \\ 74.2 & 59.7 & - & - & 3.5 \\ 89.2 & 83.1 & 2.1 & - & 38.4 \\ 90.4 & 85.0 & 1.8 & - & 29.7 \\ 90.9 & 85.8 & 1.9 & - & 30.9 \\ 76.0 & 62.5 & - & - & 8.0 \\ 76.1 & 62.6 & - & - & 28.2 \\ 76.1 & 62.7 & 1.2 & - & 4.8 \\ 89.4 & 83.5 & - & - & -\end{array}$

\section{$<2 \mu$ Fraction}

\begin{tabular}{|c|c|c|c|c|c|c|c|c|c|c|c|c|c|c|c|c|c|c|c|c|c|}
\hline 4 & $120.5-130.0$ & 121.1 & 90.4 & 84.9 & 17.6 & - & 21.6 & 20.4 & 9.1 & - & 31.3 & - & - & - & - & - & - & - & - & - & - \\
\hline 19 & $291.5-301.0$ & 292.6 & 82.3 & 72.3 & 3.4 & - & - & - & - & - & 92.0 & - & - & - & - & 4.6 & - & - & - & $\mathbf{P}$ & - \\
\hline 21 & $310.5-320.0$ & 311.2 & 93.0 & 89.1 & 8.3 & - & - & - & - & 33.6 & 56.0 & 2.1 & - & - & - & - & - & - & - & - & - \\
\hline 24 & $339.0-348.5$ & 345.9 & 92.8 & 88.7 & 6.1 & - & - & - & - & 67.9 & 22.2 & 3.8 & - & - & - & - & - & - & - & - & - \\
\hline 25 & $348.5-358.0$ & 353.9 & 80.5 & 69.5 & - & 7.4 & - & - & - & 92.6 & - & - & - & - & - & - & - & - & - & - & - \\
\hline
\end{tabular}

$\begin{array}{cccccccc}11.9 & 4.2 & 60.3 & - & - & - & - & - \\ 60.4 & - & 9.9 & - & - & 1.7 & - & - \\ 61.5 & - & - & - & 32.5 & - & 2.5 & \text { A } \\ - & - & - & - & - & 10.9 & 48.6 & - \\ - & - & - & - & - & 17.6 & 50.9 & - \\ 22.4 & - & - & - & - & 10.7 & 34.1 & - \\ 45.2 & - & 6.0 & 25.4 & - & 2.6 & 12.9 & - \\ 47.4 & - & 16.9 & - & - & 7.5 & - & - \\ 26.3 & - & - & 65.0 & - & 2.7 & - & - \\ 18.0 & - & - & 62.7 & - & - & - & -\end{array}$




\begin{tabular}{|c|c|c|c|c|c|c|c|c|c|c|c|c|c|c|c|c|c|c|c|c|c|c|c|c|}
\hline Core & $\begin{array}{c}\text { Cored } \\
\text { Interval } \\
\text { Below Sea } \\
\text { Floor (m) }\end{array}$ & $\begin{array}{l}\text { Sample Deptha } \\
\text { Below Sea } \\
\text { Floor } \\
\text { (m) }\end{array}$ & 芯 & $\begin{array}{l}\dot{2} \\
\text { : } \\
\text { छे }\end{array}$ & లु & 亗 & 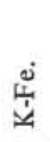 & $\stackrel{\stackrel{\Xi}{0}}{2}$ & 递 & 룔 & 总 & 茂 & $\vec{a}$ & 兄 & 胥 & 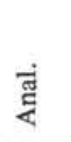 & 完 & 롱 & 嶽 & 苞 & $\begin{array}{l}\text { हే } \\
\text { हే }\end{array}$ & $\frac{0}{5}$ & $\begin{array}{l}i \\
\dot{n}\end{array}$ & $\begin{array}{l}\tilde{D} \\
\dot{D}\end{array}$ \\
\hline \multicolumn{25}{|c|}{$<2 \mu \mathrm{m}$ Fraction - Continued } \\
\hline 26 & $358.0-367.5$ & 360.2 & 81.9 & 71.7 & & - & & - & - & - & - & 91.9 & - & - & - & 8.1 & - & - & - & - & $\mathrm{T}$ & - & - & \\
\hline 30 & $396.0-405.5$ & 396.9 & 93.4 & 89.7 & & - & & 24.2 & - & - & - & 47.2 & - & - & - & - & - & - & 28.6 & - & - & - & - & \\
\hline 31 & $405.5-415.0$ & 406.6 & 90.6 & 85.3 & & - & & 12.7 & - & - & - & 47.2 & - & - & - & - & 16.5 & - & 23.6 & - & - & - & - & \\
\hline 32 & $415.0-424.5$ & 418.0 & 91.6 & 86.8 & & - & & 7.5 & - & - & - & 80.7 & - & - & - & - & 11.8 & - & - & - & - & - & - & \\
\hline 33 & $424.5-434.0$ & 425.5 & 82.4 & 72.6 & & - & & 3.9 & - & - & - & 89.1 & - & - & 5.7 & - & 1.4 & - & - & - & - & - & $\mathrm{T}$ & \\
\hline \multirow[t]{3}{*}{34} & $434.0-443.5$ & 434.5 & 80.1 & 68.9 & & - & & 6.9 & - & - & - & 90.3 & - & - & - & - & 2.0 & - & - & 0.8 & - & - & $\mathrm{T}$ & \\
\hline & & 439.2 & 81.2 & 70.7 & & - & & - & - & - & - & 65.2 & - & - & 29.8 & - & 2.1 & - & - & 2.9 & - & - & - & \\
\hline & & 439.2 & 87.0 & 79.6 & & - & & - & - & 67.0 & - & 22.6 & - & - & 10.4 & - & - & - & - & - & - & - & - & \\
\hline
\end{tabular}

aChab $=$ Chabazite. $\mathrm{P}=$ present $; \mathrm{T}=$ trace $; \mathrm{A}=$ abundant. bU-4 peaks at $6.89 \AA, 8.25 \AA$, and $3.07 \AA$, among others. cU-5 peak at $3.45 \AA$ (broad).

dU-6 peaks at $3.63 \AA, 3.08 \AA$, and $2.04 \AA$, among others. 
TABLE 15

Results of X-Ray Diffraction Analysis from Hole 217

\begin{tabular}{|c|c|c|c|c|c|c|c|c|c|c|c|c|c|c|c|c|}
\hline Core & $\begin{array}{c}\text { Cored } \\
\text { Interval } \\
\text { Below Sea } \\
\text { Floor }(\mathrm{m})\end{array}$ & $\begin{array}{l}\text { Sample Deptha } \\
\text { Below Sea } \\
\text { Floor } \\
\text { (m) }\end{array}$ & 㝕 & $\begin{array}{l}\dot{0} \\
\text { 号 } \\
\text { 定 }\end{array}$ & गु & $\stackrel{\dot{\circ}}{\circ}$ & 岂 & $\frac{0}{4}$ & 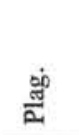 & 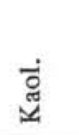 & હٍ่ & 这 & $\stackrel{\stackrel{+}{\check{c}}}{\Sigma}$ & $\dot{\Xi}$ & 离 & 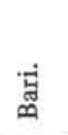 \\
\hline \multicolumn{17}{|c|}{ Bulk Samples } \\
\hline 1 & $0.0-9.5$ & 0.4 & 67.2 & 48.7 & 68.8 & - & 11.1 & & 2.9 & 2.2 & 13.7 & 1.3 & - & & & \\
\hline 2 & $40.0-49.5$ & 43.7 & 61.4 & 39.7 & 87.6 & - & 4.1 & & 1.1 & - & 6.0 & - & 1.1 & & & \\
\hline 4 & $116.0-125.5$ & 122.6 & 64.0 & 43.8 & 86.3 & - & 3.7 & & 1.2 & 2.1 & 3.5 & - & 3.2 & & & \\
\hline 34 & $582.0-591.5$ & 582.5 & 60.2 & 37.8 & 85.2 & - & - & & - & - & - & - & 14.8 & & & \\
\hline 37 & $610.5-614.5$ & 611.8 & 57.5 & 33.6 & 12.1 & 80.0 & 0.5 & & - & - & - & - & 7.5 & & & \\
\hline \multicolumn{17}{|c|}{ 2-20 $\mu \mathrm{m}$ Fraction } \\
\hline 2 & $40.0-49.5$ & 43.7 & 78.1 & 65.8 & & & 47.0 & 3.9 & 15.6 & 4.1 & 26.8 & 2.6 & - & - & - & - \\
\hline 34 & $582.0-591.5$ & 582.5 & 79.7 & 68.2 & & & 2.5 & - & - & - & 4.9 & - & 75.4 & 6.8 & 5.6 & 4.8 \\
\hline 37 & $610.5-614.5$ & 611.8 & 99.0 & 98.4 & & & 27.3 & - & - & - & - & - & - & 31.7 & 19.6 & 21.3 \\
\hline \multicolumn{17}{|c|}{$<2 \mu$ m Fraction } \\
\hline 1 & $0.0-9.5$ & 0.4 & 82.7 & 73.0 & & & 19.1 & 2.2 & 2.9 & 4.4 & 37.8 & 7.3 & 26.4 & - & - & - \\
\hline 2 & $.40 .0-49.5$ & 43.7 & 78.4 & 66.2 & & & 16.0 & - & 1.8 & 10.1 & 31.2 & 5.0 & 35.9 & - & - & - \\
\hline 4 & $116.0-125.5$ & 122.6 & 79.5 & 67.9 & & & 11.0 & 1.8 & 1.1 & 11.4 & 23.1 & 3.6 & 48.0 & - & - & - \\
\hline 34 & 582.0-591.5 & 582.5 & 78.8 & 66.8 & & & 0.7 & - & - & - & - & - & 91.8 & 2.0 & 2.4 & 3.1 \\
\hline 37 & $610.5-614.5$ & 611.8 & 81.3 & 70.8 & & & 6.5 & - & - & - & 4.3 & - & 81.1 & 5.4 & 2.7 & - \\
\hline
\end{tabular}


TABLE 16

Results of X-Ray Diffraction Analysis from Hole 218

\begin{tabular}{|c|c|c|c|c|c|c|c|c|c|c|c|c|c|c|c|}
\hline Core & $\begin{array}{c}\text { Cored } \\
\text { Interval } \\
\text { Below Sea } \\
\text { Floor }(\mathrm{m})\end{array}$ & $\begin{array}{l}\text { Sample Deptha } \\
\text { Below Sea } \\
\text { Floor } \\
(\mathrm{m})\end{array}$ & 巡 & $\begin{array}{l}\dot{0} \\
\text { 官 } \\
\text { 安 }\end{array}$ & ల్ & @̊ & ஜ্் & 苞 & 造 & $\frac{\pi}{20}$ & $\begin{array}{l}\text { ¿ } \\
\ddot{\Xi}\end{array}$ & 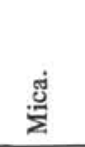 & हू் & 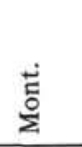 & 苞 \\
\hline \multicolumn{16}{|c|}{ Bulk Sample } \\
\hline \multirow[t]{2}{*}{2} & $4.0-13.5$ & 5.3 & 73.9 & 59.3 & 8.1 & - & - & 21.1 & 2.7 & 6.7 & - & 55.5 & 4.8 & - & 1.1 \\
\hline & & 8.2 & 76.6 & 63.4 & - & - & - & 19.4 & 3.2 & 6.4 & - & 65.2 & 5.6 & - & - \\
\hline 5 & $70.0-79.5$ & 71.0 & 75.6 & 61.9 & - & - & - & 25.7 & - & 5.8 & - & 60.8 & 7.8 & - & - \\
\hline 6 & $108.0-117.5$ & 109.7 & 74.6 & 60.3 & 49.7 & - & - & 14.2 & - & 3.1 & 2.5 & 20.5 & 2.5 & 7.5 & - \\
\hline 8 & $184.0-193.5$ & 186.1 & 77.8 & 65.4 & 6.8 & - & - & 21.6 & - & 4.4 & 3.1 & 53.1 & 6.1 & 4.8 & - \\
\hline 13 & $374.0-383.5$ & 376.6 & 66.1 & 47.0 & 8.2 & 1.9 & - & 23.8 & 3.3 & 7.8 & - & 49.0 & 4.6 & 1.5 & - \\
\hline 15 & $450.0-459.5$ & 451.0 & 70.8 & 54.4 & 4.2 & 1.9 & - & 20.8 & - & 3.2 & - & 62.7 & 7.3 & - & - \\
\hline 16 & $459.5-469.0$ & 460.9 & 70.5 & 54.0 & 3.9 & 1.7 & - & 21.6 & 2.1 & 5.9 & - & 56.5 & 5.8 & 2.5 & - \\
\hline 17 & $469.0-478.5$ & 470.4 & 73.4 & 58.4 & 3.9 & 2.3 & - & 24.1 & - & 5.6 & 3.0 & 52.7 & 6.7 & 1.7 & - \\
\hline 19 & $488.0-497.5$ & 489.4 & 72.3 & 56.7 & 4.2 & 1.6 & 2.1 & 24.2 & 3.2 & 4.8 & 2.1 & 50.9 & 5.9 & 0.9 & - \\
\hline 20 & $497.5-507.0$ & 497.9 & 70.7 & 54.3 & 3.2 & - & - & 26.8 & 2.5 & 4.9 & - & 52.3 & 7.8 & 2.5 & - \\
\hline 21 & $535.5-545.0$ & 536.4 & 64.5 & 44.5 & 5.2 & - & - & 25.6 & 2.4 & 6.2 & - & 52.2 & 7.1 & 1.2 & - \\
\hline 22 & $573.5-583.0$ & 575.7 & 69.5 & 52.4 & 4.7 & 2.0 & - & 25.4 & 2.9 & 6.3 & - & 51.4 & 7.2 & - & - \\
\hline \multirow[t]{2}{*}{23} & $611.5-621.0$ & 611.7 & 71.2 & 55.0 & 3.7 & 2.6 & - & 23.4 & 2.7 & 6.1 & - & 51.6 & 8.4 & 1.3 & - \\
\hline & & 613.8 & 74.0 & 59.3 & - & - & - & 21.0 & 3.0 & 7.5 & 5.8 & 27.0 & 4.3 & 31.5 & - \\
\hline 24 & $649.5-659.0$ & 651.8 & 59.8 & 37.2 & 3.1 & 1.3 & - & 30.4 & 3.5 & 10.7 & - & 46.9 & 4.1 & - & - \\
\hline 25 & $687.5-697.0$ & 690.0 & 64.2 & 44.1 & 5.3 & 3.0 & - & 27.4 & 3.0 & 8.3 & - & 45.6 & 5.4 & 2.1 & - \\
\hline 26 & $725.5-735.0$ & 726.7 & 67.3 & 48.9 & 6.7 & 2.3 & - & 21.6 & 2.7 & 4.4 & - & 54.3 & 8.1 & - & - \\
\hline 27 & $763.5-773.0$ & 764.8 & 62.0 & 40.7 & 6.6 & 2.2 & - & 30.6 & 3.2 & 8.2 & - & 43.9 & 5.3 & - & - \\
\hline \multicolumn{16}{|c|}{$2-20 \mu \mathrm{m}$ Fraction } \\
\hline \multirow[t]{2}{*}{2} & $4.0-13.5$ & 5.3 & 64.9 & 45.2 & & & & 35.1 & 6.0 & 13.8 & - & 38.4 & 5.4 & - & 1.4 \\
\hline & & 8.2 & 63.9 & 43.6 & & & & 27.3 & 4.6 & 11.1 & - & 49.8 & 6.0 & - & 1.1 \\
\hline 5 & $70.0-79.5$ & 71.0 & 62.2 & 40.9 & & & & 35.4 & 2.1 & 9.8 & - & 46.0 & 6.8 & - & - \\
\hline 6 & $108.0-117.5$ & 109.7 & 70.7 & 54.2 & & & & 46.1 & 3.7 & 14.6 & 1.4 & 29.5 & 4.8 & - & - \\
\hline 8 & $184.0-193.5$ & 186.1 & 68.2 & 50.3 & & & & 40.5 & 3.3 & 12.6 & - & 37.8 & 5.7 & - & - \\
\hline 13 & $374.0-383.5$ & 376.6 & 56.8 & 32.5 & & & & 39.2 & 5.7 & 13.3 & - & 37.3 & 4.5 & - & - \\
\hline 15 & $450.0-459.5$ & 451.0 & 58.2 & 34.7 & & & & 28.5 & 2.4 & 7.0 & - & 54.9 & 7.3 & - & - \\
\hline 16 & $459.5-469.0$ & 460.9 & 52.7 & 26.1 & & & & 34.3 & 2.9 & 9.2 & - & 46.5 & 7.2 & - & - \\
\hline 17 & $469.0-478.5$ & 470.4 & 59.0 & 36.0 & & & & 32.2 & 3.3 & 8.5 & - & 48.1 & 7.8 & - & - \\
\hline 19 & $488.0-497.5$ & 489.4 & 65.4 & 45.9 & & & & 33.3 & 3.5 & 6.9 & - & 48.6 & 7.8 & - & - \\
\hline 20 & $497.5-507.0$ & 497.9 & 58.7 & 35.5 & & & & 34.0 & 2.3 & 11.3 & - & 45.4 & 7.0 & - & - \\
\hline 21 & $535.5-545.0$ & 536.4 & 57.6 & 33.8 & & & & 41.1 & 3.7 & 12.4 & - & 38.2 & 4.6 & - & - \\
\hline 22 & $573.5-583.0$ & 575.7 & 53.0 & 26.5 & & & & 36.6 & 2.1 & 14.1 & - & 40.7 & 6.4 & - & - \\
\hline \multirow[t]{2}{*}{23} & $611.5-621.0$ & 611.7 & 58.7 & 35.5 & & & & 29.4 & 3.2 & 8.6 & - & 50.8 & 8.0 & - & - \\
\hline & & 613.8 & 76.5 & 63.2 & & & & 34.7 & 5.3 & 11.6 & 2.1 & 32.6 & 3.7 & 10.1 & - \\
\hline 24 & $649.5-659.0$ & 651.8 & 54.1 & 28.3 & & & & 31.2 & 2.1 & 8.7 & - & 51.7 & 6.4 & - & - \\
\hline 25 & $687.5-697.0$ & 690.0 & 54.5 & 28.9 & & & & 33.3 & 2.7 & 9.8 & - & 47.3 & 6.9 & - & - \\
\hline
\end{tabular}


TABLE 16 - Continued

\begin{tabular}{|c|c|c|c|c|c|c|c|c|c|c|c|c|c|c|c|}
\hline Core & $\begin{array}{l}\text { Cored } \\
\text { Interval } \\
\text { Below Sea } \\
\text { Floor (m) }\end{array}$ & $\begin{array}{l}\text { Sample Deptha } \\
\text { Below Sea } \\
\text { Floor } \\
\text { (m) }\end{array}$ & 产 & 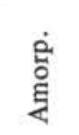 & లु & $\stackrel{\circ}{\circ}$ & ரृ & है & 它 & $\frac{a j}{20}$ & 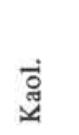 & 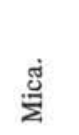 & 을 & $\stackrel{\vec{E}}{\dot{E}}$ & 完 \\
\hline \multicolumn{16}{|c|}{$<2 \mu \mathrm{m}$ Fraction } \\
\hline \multirow[t]{2}{*}{2} & $4.0-13.5$ & 5.3 & 79.4 & 67.9 & & & & 18.4 & - & 3.5 & 1.4 & 49.6 & 8.3 & 18.8 & \\
\hline & & 8.2 & 80.5 & 69.5 & & & & 17.6 & 2.1 & 5.7 & 2.6 & 57.9 & 7.2 & 6.8 & \\
\hline 5 & $70.0-79.5$ & 71.0 & 80.9 & 70.2 & & & & 20.6 & - & 2.0 & - & 54.3 & 11.8 & 11.3 & \\
\hline 6 & $108.0-117.5$ & 109.7 & 83.0 & 73.4 & & & & 16.7 & - & 2.9 & 6.9 & 26.5 & 7.1 & 39.8 & \\
\hline 8 & $184.0-193.5$ & 186.1 & 79.3 & 67.6 & & & & 16.9 & - & 3.7 & 3.0 & 42.1 & 9.1 & 25.3 & \\
\hline 13 & $374.0-383.5$ & 376.6 & 74.0 & 59.4 & & & & 15.2 & - & 0.4 & 5.1 & 44.6 & 10.0 & 24.7 & \\
\hline 15 & $450.0-459.5$ & 451.0 & 77.4 & 64.6 & & & & 19.8 & 2.0 & 2.2 & 2.5 & 53.1 & 8.6 & 11.7 & \\
\hline 16 & $459.5-469.0$ & 460.9 & 76.5 & 63.2 & & & & 17.9 & - & 1.7 & 1.8 & 55.6 & 9.0 & 14.0 & \\
\hline 17 & $469.0-478.5$ & 470.4 & 76.7 & 63.6 & & & & 22.8 & - & 4.0 & 1.1 & 53.0 & 9.5 & 9.7 & \\
\hline 19 & $488.0-497.5$ & 489.4 & 78.8 & 66.9 & & & & 23.4 & 1.9 & 3.9 & 3.6 & 48.5 & 7.4 & 11.3 & \\
\hline 20 & $497.5-507.0$ & 497.9 & 75.6 & 61.9 & & & & 21.7 & 2.0 & 2.5 & 3.8 & 52.0 & 8.5 & 9.4 & \\
\hline 21 & $535.5-545.0$ & 536.4 & 71.7 & 55.7 & & & & 16.0 & 1.8 & 1.4 & 5.5 & 55.2 & 7.3 & 12.9 & \\
\hline 22 & $573.5-583.0$ & 575.7 & 76.1 & 62.6 & & & & 18.5 & 2.2 & 3.1 & 4.7 & 49.8 & 8.1 & 13.6 & \\
\hline \multirow[t]{2}{*}{23} & $611.5-621.0$ & 611.7 & 77.2 & 64.4 & & & & 21.0 & 3.2 & 2.9 & 4.8 & 49.3 & 7.3 & 11.5 & \\
\hline & & 613.8 & 77.5 & 64.8 & & & & 7.9 & 2.8 & 1.7 & 10.6 & 8.1 & 3.3 & 65.5 & \\
\hline 24 & $649.5-659.0$ & 651.8 & 71.1 & 54.9 & & & & 16.1 & - & 0.9 & - & 55.5 & 11.6 & 15.8 & \\
\hline 25 & $687.5-697.0$ & 690.0 & 71.9 & 56.2 & & & & 16.1 & - & 1.5 & - & 49.7 & 9.4 & 23.4 & \\
\hline \multicolumn{16}{|c|}{ Interstitial Water Samples } \\
\hline 2 & $4.0-13.5$ & 8.9 & 71.3 & 55.1 & 11.9 & - & - & 20.7 & 2.7 & 7.9 & 1.3 & 50.1 & 3.8 & 1.5 & \\
\hline 3 & $13.5-23.0$ & 15.1 & 65.8 & 46.5 & - & - & - & 23.9 & 3.1 & 8.2 & - & 59.6 & 5.2 & - & \\
\hline 4 & $41.5-51.0$ & 43.3 & 69.8 & 52.8 & - & 0.9 & - & 22.9 & 3.3 & 6.6 & - & 61.4 & 4.9 & - & \\
\hline 5 & $70.0-79.5$ & 71.9 & 69.3 & 52.1 & - & 0.7 & - & 22.4 & 4.1 & 7.0 & - & 60.1 & 5.7 & - & \\
\hline 6 & $108.0-117.5$ & 109.0 & 63.0 & 42.1 & 87.1 & - & - & 4.0 & - & 1.0 & 1.7 & 3.9 & 0.5 & 1.8 & \\
\hline 8 & $184.0-193.5$ & 187.3 & 72.6 & 57.1 & 24.0 & - & - & 29.5 & 2.8 & 9.7 & 1.8 & 23.9 & 4.1 & 4.3 & \\
\hline 9 & $222.0-231.5$ & 231.5 & 72.8 & 57.5 & - & 1.8 & - & 26.0 & 2.8 & 6.5 & 1.4 & 54.8 & 6.6 & - & \\
\hline 11 & $298.0-307.5$ & 299.8 & 55.6 & 30.6 & - & 1.1 & - & 36.7 & 11.9 & 14.0 & - & 33.8 & 2.5 & - & \\
\hline 13 & $374.0-383.5$ & 375.8 & 66.9 & 48.3 & 46.2 & - & - & 11.8 & 1.5 & 1.6 & 1.3 & 29.8 & 2.3 & 5.4 & \\
\hline 14 & $412.0-421.5$ & 421.5 & 65.7 & 46.4 & 24.4 & 1.4 & - & 18.7 & 2.3 & 4.7 & - & 41.8 & 5.1 & 1.6 & \\
\hline 15 & $450.0-459.5$ & 450.2 & 63.1 & 42.3 & 6.0 & 1.9 & - & 22.3 & 2.9 & 4.5 & - & 54.7 & 7.7 & - & \\
\hline 16 & $459.5-469.0$ & 460.3 & 60.7 & 38.6 & 4.4 & 1.4 & - & 30.3 & 3.5 & 11.3 & - & 45.4 & 3.7 & - & \\
\hline 18 & $478.5-488.0$ & 488.0 & 67.6 & 49.4 & 13.7 & 1.8 & 3.9 & 23.3 & - & 5.8 & - & 45.0 & 5.3 & 1.3 & \\
\hline 21 & $535.5-545.0$ & 538.8 & 64.5 & 44.5 & 5.5 & 2.2 & - & 24.9 & 1.5 & 6.1 & - & 53.2 & 6.6 & - & \\
\hline 22 & $573.5-583.0$ & 575.3 & 58.9 & 35.8 & 5.0 & 2.3 & - & 30.6 & 3.3 & 12.2 & - & 42.2 & 4.3 & - & \\
\hline 23 & $611.5-621.0$ & 613.3 & 71.4 & 55.3 & 5.6 & 1.5 & - & 22.7 & 2.3 & 4.6 & 1.9 & 46.3 & 5.7 & 9.3 & \\
\hline 24 & $649.5-659.0$ & 651.3 & 72.4 & 57.0 & 3.8 & 1.1 & - & 24.3 & 2.4 & 6.3 & 2.9 & 39.4 & 5.9 & 13.8 & \\
\hline 25 & $687.5-697.0$ & 689.3 & 63.1 & 42.3 & 1.3 & 1.5 & - & 33.0 & 1.8 & 9.2 & - & 47.6 & 5.6 & - & \\
\hline 26 & $725.5-735.0$ & 727.3 & 63.9 & 43.5 & 6.2 & 2.4 & - & 31.0 & 3.2 & 11.9 & - & 40.6 & 4.7 & - & \\
\hline 27 & $763.5-773.0$ & 765.3 & 71.8 & 55.9 & 5.1 & 2.6 & - & 24.6 & 2.3 & 5.5 & - & 50.4 & 8.0 & 1.5 & \\
\hline
\end{tabular}


J. MATTI, I. ZEMMELS, H. E. COOK

TABLE 17

Sample Positions for Site 218 Interstitial Water Analysis

\begin{tabular}{ccc}
\hline Core/Section & $\begin{array}{c}\text { Depth in } \\
\text { Section (cm) }\end{array}$ & $\begin{array}{c}\text { Depth Below } \\
\text { Sea Floor (m) }\end{array}$ \\
\hline $2-4$ & $0-20$ & 8.9 \\
$3-2$ & $0-10$ & 15.1 \\
$4-2$ & $20-30$ & 43.3 \\
$5-2$ & $20-40$ & 71.9 \\
$6-1$ & $104-110$ & 109.0 \\
$8-3$ & $0-20$ & 187.3 \\
9, CC & - & 231.5 \\
$11-2$ & $20-30$ & 299.8 \\
$13-2$ & $20-30$ & 375.8 \\
14, CC & - & 421.5 \\
$15-1$ & $20-27$ & 450.2 \\
$16-2$ & $20-30$ & 460.3 \\
18, CC & - & 488.0 \\
$21-3$ & $0-20$ & 538.8 \\
$22-2$ & $20-30$ & 575.3 \\
$23-2$ & $20-30$ & 613.3 \\
$24-2$ & $20-30$ & 651.3 \\
$25-2$ & $20-30$ & 689.3 \\
$26-2$ & $20-30$ & 727.3 \\
$27-2$ & $20-30$ & 765.3 \\
\hline
\end{tabular}

